\title{
Supporting Information: DICE: A Monte Carlo code for molecular simulation including Configurational Bias Monte Carlo method
}

\author{
Henrique Musseli Cezar; Sylvio Canuto†, Kaline Coutinho \\ Universidade de Sao Paulo, Instituto de Fisica, 05508-090, Sao Paulo, SP, Brazil
}

\section{SI 1: DICE implementation and features}

\section{SI 1.1: Overview}

DICE is a software for the simulation of gases and liquids using Monte Carlo methods, as described in main text, written in Fortran using OpenMP for a shared memory parallelization. This code is available at https://portal. if .usp.br/dice. The units of the variables are: $\mathrm{kcal} / \mathrm{mol}$ for energy, $\AA$ for distance and positions, $\mathrm{K}$ for temperature, atm for pressure, $\mathrm{g} / \mathrm{cm}^{3}$ for density, $e$ for charge, ${ }^{\circ}$ for angles and $\mathrm{kcal} /\left(\mathrm{mol} \AA^{2}\right)$ and $\mathrm{kcal} /\left(\mathrm{mol} \mathrm{rad}^{2}\right)$ for spring constants for the bonds and angles, respectively. DICE uses the Mersenne Twister pseudo random number generator,[1] specifically, the implementation by Tsuyoshi Tada.[2] Along this text, important input keywords will be presented in parenthesis. The current version of the software is able to do simulations in the NVT (vstep $=0$ ) and NPT ensembles, in periodic boxes with two shapes: cubic (geom $=0$, default) or parallelepiped (geom $=1$ ). The initial configuration can be read or randomly generated, with the minimum distance between atoms defined by the user (rmin_ini $=1.5$, default). During the run, the state of the simulation is saved periodically (isave $=1000$, default), so the user may continue $($ accum $=$ yes $)$ a simulation.

A simple Verlet neighbor list is used to reduce computational cost.[3] In this scheme, additionally to the usual cutoff radius, $r_{\text {cut }}$, a buffer radius, $r_{\text {buf }}$, is used to keep track of neighbor molecules that might interact, not including in the neighbor list molecules that are further than $r_{\text {buf }}$. As default options, the $r_{\text {buf }}$ is the half of the minimum length size of the box (rbuf $=L_{\text {min }} / 2$, default) and the $r_{\text {cut }}$ is $92 \%$ of the $r_{\text {buf }}\left(\right.$ rcut $=0.92 r_{\text {buf }}$, default $)$. This length of $8 \%$ for the $r_{\text {buf }}$ was suggested in literature[3] as an optimum value for liquid systems, but it might be modified by the user to obtain a good compromise between the

\footnotetext{
*henrique.cezar@usp.br

†canuto@if.usp.br

${ }^{\ddagger}$ kaline@if.usp.br
} 
number of steps needed between each neighbor list update and an effective reduction in the number of possible interacting molecules. The default update of the neighbor list is calculated based in simulations of liquid water at room conditions in different size boxes with default values of $r_{\text {cut }}$ and $r_{\text {buf }}$, as upbuf $=\operatorname{int}\left(\left(r_{\text {buf }}-r_{\text {cut }}\right) / 0.013\right)$. As examples, for simulations with $N=500,1000$ and 3000 water molecules, the upbuf $=75,95$ and 137, respectively.

Several default values are provided for the algorithmic options and parameters, and the automatic adjustment of parameters is done for some cases. The goal is to keep the simple input file for the user, but also allow the user to have control over the simulation. Even though the main focus is solute-solvent systems, the software tries to be general so different systems can be simulated.

\section{SI 1.2: Initialization and Metropolis MC}

The algorithm implemented in the DICE starts by performing the initialization of the variables, reading the information from input files. At least three files are read (see Sec. SI 5 for examples): (i) a file with keywords information about the simulated ensemble, thermodynamic conditions, different types of molecules and their quantities, number of steps, algorithmic options and other information (Examples: octane.ter, neopentane.txt, 4ethylheptane.txt, dce.ter and subpc.ter for thermalization stage and octane.in, dce.in and subpc.in for equilibrium stage); (ii) a file containing information of $m$ different types of molecules that compose the system. For each molecule the information are: the quantity of atoms, the identification name (namid), the initial geometry (Cartesian coordinates) and the force field non-bonded parameters for each atom: charge $q_{i}$ for the Coulomb potential and $\epsilon_{i}$ and $\sigma_{i}$ for the Lennard-Jones potential (Examples: octane.txt, dce.txt and subpc.txt); and (iii) a file containing the fragments composition and connections (\$atoms fragments and \$fragment connection blocks of data), topology and the force field bonded parameters (\$bond, \$angle, \$dihedral and \$improper blocks of data) for the flexible or semi-flexible molecules (Examples: octane.dfr, dce.dfr and subpc.dfr). Additionally, a file containing the initial configuration of the simulation may also be provided (init $=$ yesreadxyz), otherwise, the initial configuration is generated randomly (init $=$ yes).

After the initialization, the simulation starts. In the $N V T$ ensemble, a new trial configuration, or one MC step, is always generated by selecting one molecule from the box (molecule $A$ ), either randomly or with preferential sampling[4,5] (sampling $=2$ ). After selecting $N$ molecules one MC cycle is completed. In each MC step, a rigid trial (translation and rotation at the center of mass) or a flexible trial (an internal deformation with the CBMC method) is performed with a specific ratio of flexible trial (pcbmc $=0.5$, default). The flexible trial is performed only if the keyword $f l e x=$ nameid exist in the input file ${ }^{*}$.txt and, in this case, a file nameid.dfr should also exist containing the fragments information. In the ${ }^{*}$.dfr file each fragment should be identified by its set of atoms and a label that defines its status: $\mathrm{R}$ for rigid, $\mathrm{F}$ for semi-flexible (rigid bonds only) and $\mathrm{M}$ for flexible. If a fragment is rigid, the fragment library for this specific fragment is not generated. If a fragment is semi-flexible or flexible, a fragment library will be generated in the pre-simulation stage where a simple MC 
simulation of the isolated fragment is performed saving $N_{s f}$ configurations (nsf $=10000$, default) in a specific interval of steps (sfint $=500$, default).

The rigid trial is composed by a random displacement of $\delta x, \delta y$ and $\delta z$, within a selfadjusted maximum value of $\pm \delta r_{\max }$ performed in specific number of MC cycles (nratio = 50 , default), and a random rotation around one of the Cartesian axis within a maximum values of $\pm 15^{\circ}$. In the $N P T$ ensemble, additionally one trial that changes the volume of the simulation box and rescales the molecular center of mass is also performed considering an isotropic incremental factor $\delta L$ within a self-adjusted maximum value of $\pm \delta L_{\max }$ performed in specific number of MC cycles (vratio $=50$, default). This volume trial is performed in a specific interval of $\mathrm{MC}$ cycles (nstep $=5$, default). The self-adjusted maximum values, $\delta r_{\max }$ and $\delta L_{\max }$, are used to keep the acceptance ratio of new configurations around $50 \%$ (acceptance $=0.5$, default), but their are limited by an interval of $0.01 \AA$ and $2.00 \AA$.

The potential energy of this new configuration is calculated and the acceptance of the trial move is tested. For the rigid trial the Eq. 6 or 7 is used for the Metropolis sampling or for the flexible trial the Eq. 19 or 24 is used for the CBMC sampling. If accepted, the new configuration replaces the old one, otherwise the old configuration accounts as a new one, and the values of energy, square energy and others properties of the new configuration are accumulated for later obtaining the thermodynamic properties. These steps are repeated until the total number of MC cycles required by the user is achieved. In Fig. S1 a flowchart shows the algorithm implemented in DICE.

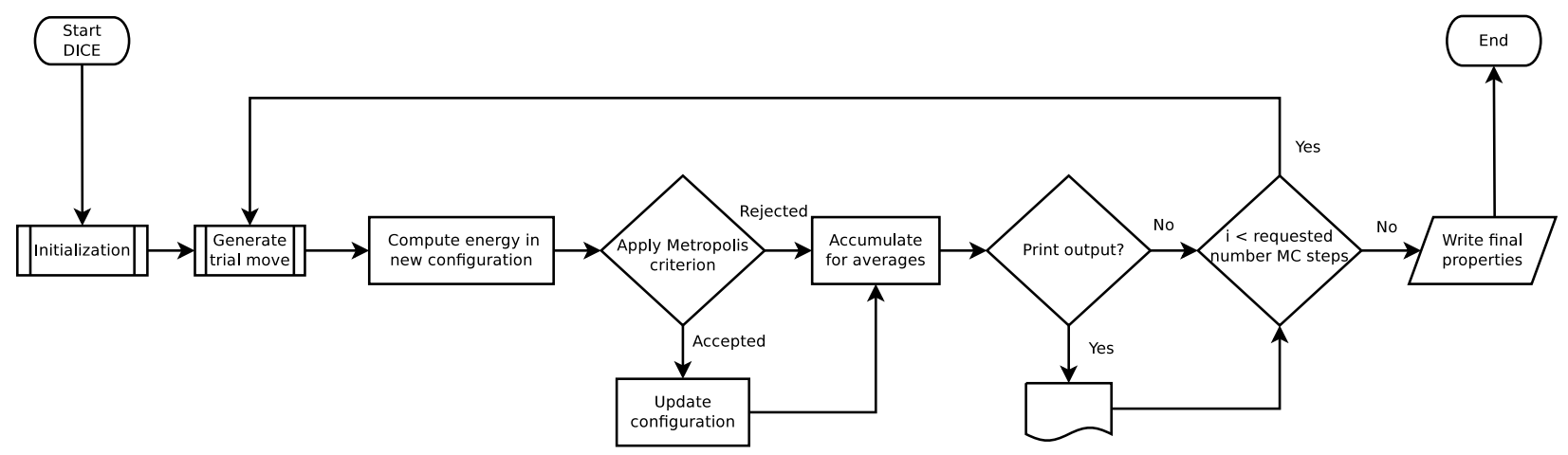

Figure S1: Flowchart of the Metropolis MC algorithm as implemented in DICE. More detail is given in the text.

The efficiency of the parallelization is measured in terms of the speedup $S=\frac{t(1)}{t(n)}$, with $t(1)$ being the walltime for the run using a single thread and $t(n)$ the walltime for the parallel run with $n$ threads. We ran simulations of 4000 and 10000 SPC/E water molecules in a box using the NPT ensemble for $1.6 \times 10^{7} \mathrm{MC}$ steps. The simulations were run in a node with two Xeon E5-2670 @ 2.60GHz and 64 GB of memory. Results are shown in Figure S2. 


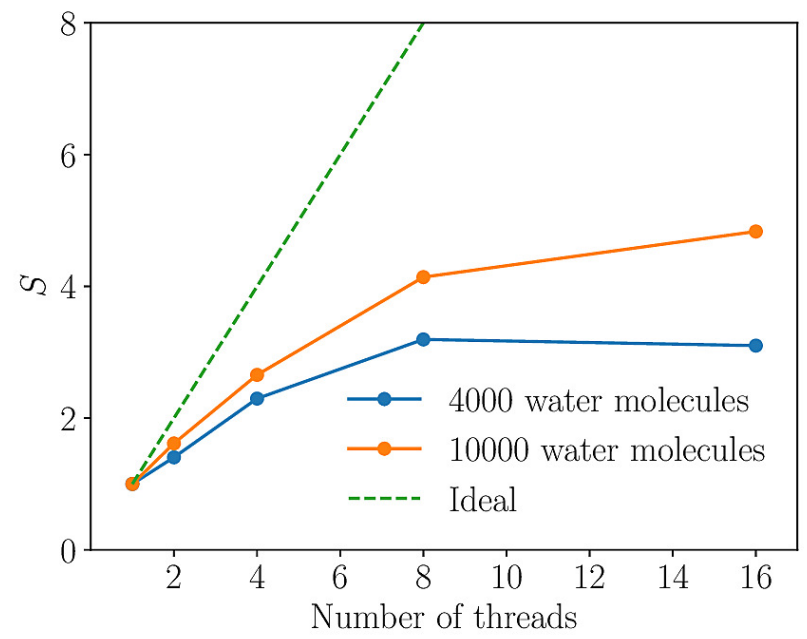

Figure S2: Speedup curve for a DICE parallel simulation in the NPT ensemble.

\section{SI 1.3: Generating the $\kappa_{\phi}$ trial insertion angles}

In DICE, the complete CBMC move consists of 5 processes:

a) breaking the flexible molecule with $N_{\text {frag }}$ fragments in a random rotatable bond, with probability $1 /\left(N_{\text {frag }}-1\right)$;

b) selecting one of the two sides and deleting the fragments $\left(N_{\text {del }}\right)$ from this side, keeping the other side unchanged. The user can select between two possibilities for this choose:

(i) with $50 \%$ of chance for each side (sweight $=$ no), or

(ii) with a weight that favors smaller parts to be sampled more often (sweight = yes, default), increasing the acceptance rates of the CBMC move. This choice is weighted by the number of atoms, and the side with less atoms is chosen more frequently with probability $1-\left(N_{\text {smaller }} / N\right)$, where $N_{\text {smaller }}$ is the number of atoms of the size with less atoms and $N$ the total number of atoms of the molecule;

c) selecting one configuration of each deleted fragment library with equal probability $1 / N_{s f}$

d) reconstructing the entire molecule connecting each fragment in the new configuration with a new dihedral angles selected with a probability proportional to the Boltzmann distribution in a small set of trial dihedral angles $\kappa_{\phi}$. Some option were implemented for this selection and will be discussed below; and

e) calculating the probability of reconstructing the old conformation of the entire molecule with a similar procedure to obtain the acceptance criterion. In doing so, the old fragment configuration and the old dihedral angles should be selected. 
The user can select between three different algorithms to select the trial insertion dihedral angles: (i) random $\phi$ sampling; (ii) equidistant $\phi$ sampling; and (iii) bias $\phi$ sampling. The first two sampling are random $($ biasphi $=$ no, default) and preliminary tests indicate that both algorithms give similar results. The random $\phi$ sampling selects the $\kappa_{\phi}$ angles (ntrialphi $=16$, default) by drawing random values in the interval $[0,2 \pi)$ (equiphi $=$ no, default). The equidistant $\phi$ sampling draws only one random angle in the same interval and then generates the other $\kappa_{\phi}-1$ trial angles by distributing the points uniformly in the circumference (equiphi $=$ yes) with the same $\Delta \phi$ value. Both algorithms make no assumption on the energy scales of the torsional and nonbonded energies.

When the minima of the torsional potential, have their positions close to the minima of the total potential energy, it is possible to improve the CBMC acceptance rate by introducing another bias. This additional bias is used while generating the $\kappa_{\phi}$ trial insertion angles, favoring dihedral angles that results in lower torsional energies (biasphi $=$ yes). We implemented this bias using the rejection method, $[6,7]$ accepting new angles with the probability $\exp \left[-\beta u_{i \alpha}^{\mathrm{dih}}(n)\right]$, where $u_{i \alpha}^{\mathrm{dih}}(n)$ is the summation of all the torsional energies of the dihedral angles that unite the fragment $i$ with the previous fragment. Introducing this bias removes the torsional energy from the $P_{\text {soft }}$ probability of Eq. 14, leaving only the nonbonded energy to be used in the CBMC bias. Following the same steps, a Rosenbluth factor that does not depend on the torsional energy is obtained, and the same acceptance criterion of Eq. 19 can be used but with the different Rosenbluth factor definition, which contains only the nonbonded energy.

\section{SI 1.4: $\quad$ Force Field}

In this version, DICE supports both OPLS[8] and AMBER[9] force fields to evaluate the energy. These force fields use simple harmonic functions to describe the bonded terms of the potential energy: bonds, angles and improper dihedral angles

$$
\begin{aligned}
\mathcal{U}_{\text {bond }} & =\sum_{i}^{\text {bonds }} K_{\mathrm{r}}^{i}\left(r_{i}-r_{\text {eq }}\right)^{2}, \\
\mathcal{U}_{\text {angle }} & =\sum_{i}^{\text {angles }} K_{\theta}^{i}\left(\theta_{i}-\theta_{\text {eq }}\right)^{2}, \\
\mathcal{U}_{\text {improper }} & =\sum_{i}^{\text {improper }} K_{\phi}^{i}\left(\phi_{i}-\phi_{\text {eq }}\right)^{2},
\end{aligned}
$$

and also cosine functions for the improper dihedral angles

$$
\mathcal{U}_{\text {improper }}=\sum_{i}^{\text {improper }} \frac{V_{2}^{i}}{2}\left[1+\cos \left(2 \phi_{i}-f_{2}^{i}\right)\right] .
$$


For the proper dihedral (torsional) angles, two forms were implemented with a slightly different Fourier terms to describe the

$$
\begin{array}{r}
\mathcal{U}_{\text {torsion }}^{\text {OPLS }}=\sum_{i}^{\text {dihedral }} \frac{V_{1}^{i}}{2}\left[1+\cos \left(\phi_{i}+f_{1}^{i}\right)\right] \\
+\frac{V_{2}^{i}}{2}\left[1-\cos \left(2 \phi_{i}+f_{2}^{i}\right)\right] \\
+\frac{V_{3}^{i}}{2}\left[1+\cos \left(3 \phi_{i}+f_{3}^{i}\right)\right] \\
+\frac{V_{4}^{i}}{2}\left[1-\cos \left(4 \phi_{i}+f_{4}^{i}\right)\right]
\end{array}
$$

and

$$
\begin{array}{r}
\mathcal{U}_{\text {torsion }}^{\text {AMBER }}=\sum_{i}^{\text {dihedral }} \frac{V_{1}^{i}}{2}\left[1+\cos \left(\phi_{i}-f_{1}^{i}\right)\right] \\
+\frac{V_{2}^{i}}{2}\left[1+\cos \left(2 \phi_{i}-f_{2}^{i}\right)\right] \\
+\frac{V_{3}^{i}}{2}\left[1+\cos \left(3 \phi_{i}-f_{3}^{i}\right)\right] \\
+\frac{V_{4}^{i}}{2}\left[1+\cos \left(4 \phi_{i}-f_{4}^{i}\right)\right] .
\end{array}
$$

The Lennard-Jones (LJ) and Coulomb (Q) potentials are used to describe the atomic pairwise non-bonded interactions

$$
\mathcal{U}_{i j}=\mathcal{U}_{i j}^{L J}+\mathcal{U}_{i j}^{Q}=f_{i j}^{\mathrm{LJ}} 4 \epsilon_{i j}\left[\left(\frac{\sigma_{i j}}{r_{i j}}\right)^{12}-\left(\frac{\sigma_{i j}}{r_{i j}}\right)^{6}\right]+f_{i j}^{\mathrm{Q}} \frac{q_{i} q_{j} e^{2}}{\left(4 \pi \varepsilon_{0}\right) r_{i j}}
$$

where $e^{2} /\left(4 \pi \varepsilon_{0}\right)=331.9684 \AA \mathrm{kcal} / \mathrm{mol}, \epsilon_{i j}=\sqrt{\epsilon_{i} \epsilon_{j}}, \sigma_{i j}=\sqrt{\sigma_{i} \sigma_{j}}$ or $\sigma_{i j}=\left(\sigma_{i}+\sigma_{j}\right) / 2$ depending on the input option and $\epsilon_{i}, \sigma_{i}$ and $q_{i}$ are the atomic parameters. For the intermolecular interactions, i.e. $i$ and $j$ belong to different molecules, $f_{i j}^{\mathrm{LJ}}=f_{i j}^{\mathrm{Q}}=1$. For the intramolecular interactions, i.e. $i$ and $j$ belong to the same molecule, the $f_{i j}$ assumes different values depending on the force field and also on the number of bonds that separate the atoms within the molecule. Atoms separated by one bond, 1-2, and two bonds, 1-3, interact only through the bonded terms, then $f_{i j}^{\mathrm{LJ}}=f_{i j}^{\mathrm{Q}}=0$. The atoms separated by three bond, 1-4, have a scaling factor in the non-bonded interaction: $f_{i j}^{\mathrm{Q}}=f_{i j}^{\mathrm{LJ}}=0.5$ for the OPLS force field (fudgelj $=0.5$ and fudgeclb $=0.5$ ) and $f_{i j}^{\mathrm{LJ}}=0.5$ and $f_{i j}^{\mathrm{Q}}=1 / 1.2$ for the AMBER (fudgelj $=0.5$ and fudgeclb $=0.8333$ ). For atoms separated by four bonds or more, the interactions are calculated considering only non-bonded interaction without any scaling factor, then $f_{i j}^{\mathrm{LJ}}=f_{i j}^{\mathrm{Q}}=1$.

Therefore, the total potential energy of a molecule $A$ is calculated considering all explicit interactions (intra and intermolecular) within the cutoff radius $r_{\text {cut }}$ and the long range 
correction (LRC)

$$
\mathcal{U}^{A}=\mathcal{U}_{\text {intra }}^{A}+\mathcal{U}_{\text {inter }}^{A}+\mathcal{U}_{\mathrm{LJ}-\mathrm{LRC}}^{A}+\mathcal{U}_{\mathrm{Q}-\mathrm{LRC}}^{A}
$$

where

$$
\mathcal{U}_{\text {intra }}^{A}=\mathcal{U}_{\text {bond }}+\mathcal{U}_{\text {angle }}+\mathcal{U}_{\text {torsion }}+\mathcal{U}_{\text {improper }}+\sum_{\substack{i=1 \\ i \in A}}^{N_{A}-1} \sum_{\substack{j=i+1 \\ j \in A}}^{N_{A}} \mathcal{U}_{i j}
$$

and

$$
\mathcal{U}_{\text {inter }}^{A}=\sum_{B}^{R_{A B} \leq r_{\text {cut }}} \sum_{\substack{i=1 \\ i \in A}}^{N_{A}} \sum_{\substack{j=1 \\ j \in B}}^{N_{B}} \mathcal{U}_{i j}
$$

which $B$ are the other molecules inside $r_{\text {cut }}$ and $R_{A B}$ are the center of mass distance between $A$ and $B$.

The two LRC terms are approximations to calculate the energy contribution given by molecules with $r_{\text {cut }}<R_{A B} \leq \infty$. For the Lennard-Jones potential, an uniform distribution of molecules is assumed beyond $r_{\text {cut }}$ to infinity, i.e. $g\left(r_{i j}\right) \approx 1$, then

$$
\mathcal{U}_{\mathrm{LJ}-\mathrm{LRC}}^{A}=2 \pi N \rho \sum_{B} \sum_{\substack{i=1 \\ i \in A}}^{N_{A}} \sum_{\substack{j=1 \\ j \in B}}^{N_{B}} \frac{4 \epsilon_{i j} \sigma_{i j}^{3}}{9}\left[\left(\frac{\sigma_{i j}}{r_{i j}}\right)^{9}-3\left(\frac{\sigma_{i j}}{r_{i j}}\right)^{3}\right]
$$

which $B$ are the molecule types that compose the system.

For the Coulomb potential, an dielectric continuum larger than 1.0 is assumed beyond $r_{\text {cut }}$ to infinity, generating a reaction field (dielectric $=1.0$, default, does not calculate the LRC term for the Coulomb potential). Therefore, it is necessary to define the keyword dielectric with a value larger than 1.0 to take this term into account. We implemented two models for the reaction field: (i) the model discussed by Barker and Watts[10] with the equation well presented in Ref. [11] (electrostatics = reaction-field) and (ii) the model discussed in Allen and Tildesley with the additional Born term[3] (electrostatics = reaction-field-born, default).

The first model is similar to the model implemented in other software like GROMACS

$$
\mathcal{U}_{\mathrm{Q}-\mathrm{LRC}}^{A}=\sum_{B}^{R_{A B} \leq r_{\text {cut }}} \sum_{\substack{i=1 \\ i \in A}}^{N_{A}} \sum_{\substack{j=i \\ j \in B}}^{N_{B}} q_{i} q_{j}\left(\frac{\epsilon_{\mathrm{RF}}-1}{2 \epsilon_{\mathrm{RF}}+1} \frac{r_{i j}^{2}}{r_{\text {cut }}^{3}}+\mathcal{C}\right)
$$

where $\epsilon_{\mathrm{RF}}$ is the dielectric constant of the continuum and the constant $\mathcal{C}$ is

$$
\mathcal{C}=-\frac{3 \epsilon_{\mathrm{RF}}}{2 \epsilon_{\mathrm{RF}}+1} \frac{1}{r_{\text {cut }}} .
$$

When the conformational change of molecule $A$ is performed in the CBMC step, we also take into account the self energy correction of the dielectric continuum

$$
\mathcal{U}_{\mathrm{self}}^{A}=\sum_{\substack{i=1 \\ i \in A}}^{N_{A}-1} \sum_{\substack{j=i+1 \\ j \in A}}^{N_{A}} q_{i} q_{j}\left(\frac{\epsilon_{\mathrm{RF}}-1}{2 \epsilon_{\mathrm{RF}}+1} \frac{r_{i j}^{2}}{r_{\mathrm{cut}}^{3}}+\mathcal{C}\right)
$$


This contribution is constant if the molecule is rigid and, therefore, cancels in the Metropolis acceptance criterion that considers the energy variation of the molecule $A$ in the old and new configurations, $\Delta \mathcal{U}^{A}$.

In the second model, the potential energy long range correction due to the electrostatic field on molecule $A$, considering the Born correction, is given by

$$
\mathcal{U}_{\mathrm{Q}-\mathrm{LRC}-\mathrm{RFB}}^{A}=\mathcal{U}_{\mathrm{Q}-\mathrm{LRC}}^{A}+\mathcal{U}_{\mathrm{Born}}^{A}
$$

where

$$
\mathcal{C}=\mathcal{C}_{\mathrm{RFB}}=-\frac{1}{r_{\mathrm{cut}}}\left(1+\frac{\epsilon_{\mathrm{RF}}-1}{2 \epsilon_{\mathrm{RF}}+1}-\frac{\epsilon_{\mathrm{RF}}-1}{\epsilon_{\mathrm{RF}}}\right)
$$

and

$$
\mathcal{U}_{\mathrm{Born}}^{A}=-\sum_{B}^{R_{A B} \leq r_{\mathrm{cut}}}\left(\frac{\epsilon_{\mathrm{RF}}-1}{\epsilon_{\mathrm{RF}}}\right) \frac{1}{r_{\mathrm{cut}}} Q_{A} Q_{B},
$$

with $Q_{A}$ and $Q_{B}$ being the total charges of molecules $A$ and $B$, respectively. The self polarization term is also considered with $\mathcal{C}_{\mathrm{RFB}}$ when the $\mathrm{CBMC}$ moves are performed.

\section{SI 2: n-octane}

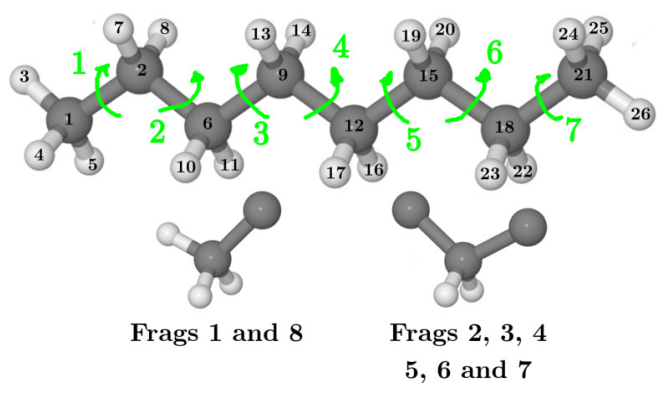

Figure S3: n-octane molecule with the atomic labels and its fragments. The green arrows and numbers indicate the rotatable bonds and its indexes.

\section{SI 2.1: Acceptance rates for different $\kappa_{\phi}$ for n-octane in gas phase}

To show the dependence of the acceptance rates and walltime with the number of trial insertion angles $\kappa_{\phi}$ we have performed simulations of n-octane in gas phase considering $\kappa_{\phi}=8,12,16,18,24,32$ and 64 and all other parameters as detailed in the main text. The simulations were run for $10^{6}$ CBMC steps under the same conditions in a desktop $\mathrm{PC}$ equipped with an Intel Core i7 3930K running at $3.2 \mathrm{GHz}$ and $64 \mathrm{~GB}$ of RAM running at $1333 \mathrm{MHz}$. The DICE OpenMP parallelization with 6 threads was employed. After the selection of the rotatable bond in each CBMC step, the side was selected considering the number of atoms attached to each extremity, favoring the side with less atoms (sweight 
= yes). This option increases the acceptance rates, as the regrowing a smaller side of the molecule with few fragments usually yields higher acceptance rates than regrowing the large side with several fragments.

In Table S1 we show the overall and partial acceptance rates (for each rotatable bond), as well as the average walltime, or the elapsed real time, for three runs. The walltime does not consider the time needed to generate the fragment library, which takes approximately $(41.2 \pm 0.3) \mathrm{s}$. Each rotatable bond was selected an equivalent number of times during the simulation, and the acceptance rates for each bond take into account the probability of accepting the CBMC move independent on which side of the rotatable bond was deleted. Therefore, the acceptance rate is an average of two rates that may be different depending on how many fragments need to be reinserted on that side. The acceptance rates vary less than $2 \%$ considering runs with different seeds.

Table S1: Acceptance rate (\%) for a simulations of n-octane with different number of trial insertion angles $\kappa_{\phi}$. The bonds are labeled according to Figure S3. The walltime was obtained as an average of 3 runs. The data shown in this table is an output of DICE in the outname . acb file.

\begin{tabular}{lccccccc}
\hline & \multicolumn{7}{c}{$\kappa_{\phi}$} \\
\cline { 2 - 8 }$\kappa_{\phi}=$ & 8 & 12 & 16 & 18 & 24 & 32 & 64 \\
\hline bond 1 & 65.9 & 68.7 & 71.8 & 72.7 & 73.9 & 75.4 & 77.6 \\
bond 2 & 33.6 & 38.1 & 43.1 & 44.4 & 47.5 & 50.8 & 56.2 \\
bond 3 & 18.8 & 22.9 & 27.9 & 29.4 & 33.0 & 37.3 & 44.2 \\
bond 4 & 14.8 & 18.6 & 23.4 & 25.2 & 29.1 & 33.8 & 41.1 \\
bond 5 & 19.3 & 23.3 & 27.9 & 29.6 & 33.7 & 38.0 & 44.1 \\
bond 6 & 33.4 & 38.2 & 42.3 & 43.3 & 46.9 & 50.0 & 55.2 \\
bond 7 & 65.7 & 69.5 & 71.6 & 72.3 & 73.9 & 75.4 & 77.4 \\
overall & 35.9 & 39.9 & 44.0 & 45.3 & 48.3 & 51.6 & 56.6 \\
\hline Walltime (s) & 86.3 & 89.4 & 103.3 & 104.9 & 120.8 & 148.3 & 222.0 \\
\hline
\end{tabular}

As we see on Table S1, by increasing the number of trial insertion angles, the acceptance rates increase, but so does the walltime. For a linear chain like n-octane, the lower acceptance rates are found in the middle of the chain, like bonds 3,4 and 5 . Indeed, the lowest acceptance rate is found for the rotatable bond 4 , which has the similar acceptance probabilities for both sides of the bond. Increasing the number of trial insertion angles from 8 to 64 , an increase of almost $27 \%$ is observed for the acceptance rate of the rotatable bond 4 . However, while the walltime increase of 2.5 times is not relevant for the gas phase simulation, it becomes important, and also more dramatic, when the solvent is considered, since for each trial insertion position the interaction with the solvent is computed. Therefore, the user should find and equilibrium between acceptance rate and computational time.

The rotatable bonds close to the extremities, like bonds 1 and 7, have a high acceptance rate independent on the number of trial insertion angles. This happens because when the 
side to be regrown is the side relative to the $\mathrm{C}_{2} \mathrm{H}_{3}$ fragment (methyl group, fragments 1 and $8)$, just one fragment needs to be inserted, and the acceptance rates for this insertion are as high as $77 \%$ for $\kappa_{\phi}=8$ and $87 \%$ for $\kappa_{\phi}=64$. Reinserting the other side of bonds 1 and 7 (which is basically regrowing the whole molecule), however, is more difficult, resulting in acceptance rates of $4 \%$ and $24 \%$ for $\kappa_{\phi}=8$ and 64 , respectively. The number shown in Table S1 is the weighted average, considering that the whole molecule is regrown only $15 \%$ of the times that the rotatable bonds 1 and 7 are selected.

\section{SI 2.2: Equilibrium constant of n-octane and rotational potential}

Assuming just three possible configurations accessible to the system: trans $(T)$, gauche $(+)$ $(G+)$ e gauche $(-)(G-)$, we have that the concentrations must sum to $100 \%$

$$
[T]+[G+]+[G-]=1
$$

Considering the symmetry of the potential among the gauche $(+)$ and gauche $(-)$ conformations, we should have equal concentrations of these two conformers:

$$
[G+]=[G-]
$$

The equilibrium constant $K$ giving the conformational changes between the trans and gauche $(+)$ conformation is then

$$
K=\frac{[G+]}{[T]}=\frac{[G+]}{1-2[G+]},
$$

from which we obtain the population of gauche+ conformations

$$
[G+]=\frac{K}{2 K+1}
$$

Since the equilibrium constant is related to the Gibbs free energy with the expression

$$
K=e^{\frac{-\Delta G}{k T}}
$$

with $k$ being the Boltzmann constant, $T$ is the temperature and $\Delta G$ is the free energy difference between the trans and gauche $(+)$ conformations. Additionally, when an isolated molecule is studied the approximation of $\Delta G \approx \Delta E-T \Delta S$ can be used considering a negligible enthalpic contributions $(P \Delta V \approx 0)$. The energy differences, $\Delta E$ are in general obtained by quantum mechanics (QM) calculations of both conformations at the optimized geometry. The vibrational contribution of entropic term, $T \Delta S$ can also be calculated by QM calculations. Therefore, the classical force field can be built considering the energetic and entropic contributions, $\Delta E_{F F}=\Delta E_{Q M}+T \Delta S_{Q M}$ or only the energetic contribution assuming a negligible entropic contribution, $\Delta E_{F F}=\Delta E_{Q M}$. Then, using the approximation of $\Delta G \approx \Delta E_{F F}$, we can finally estimate the gauche $(+)$ population, determining the gauche $(-)$ and trans populations too. 

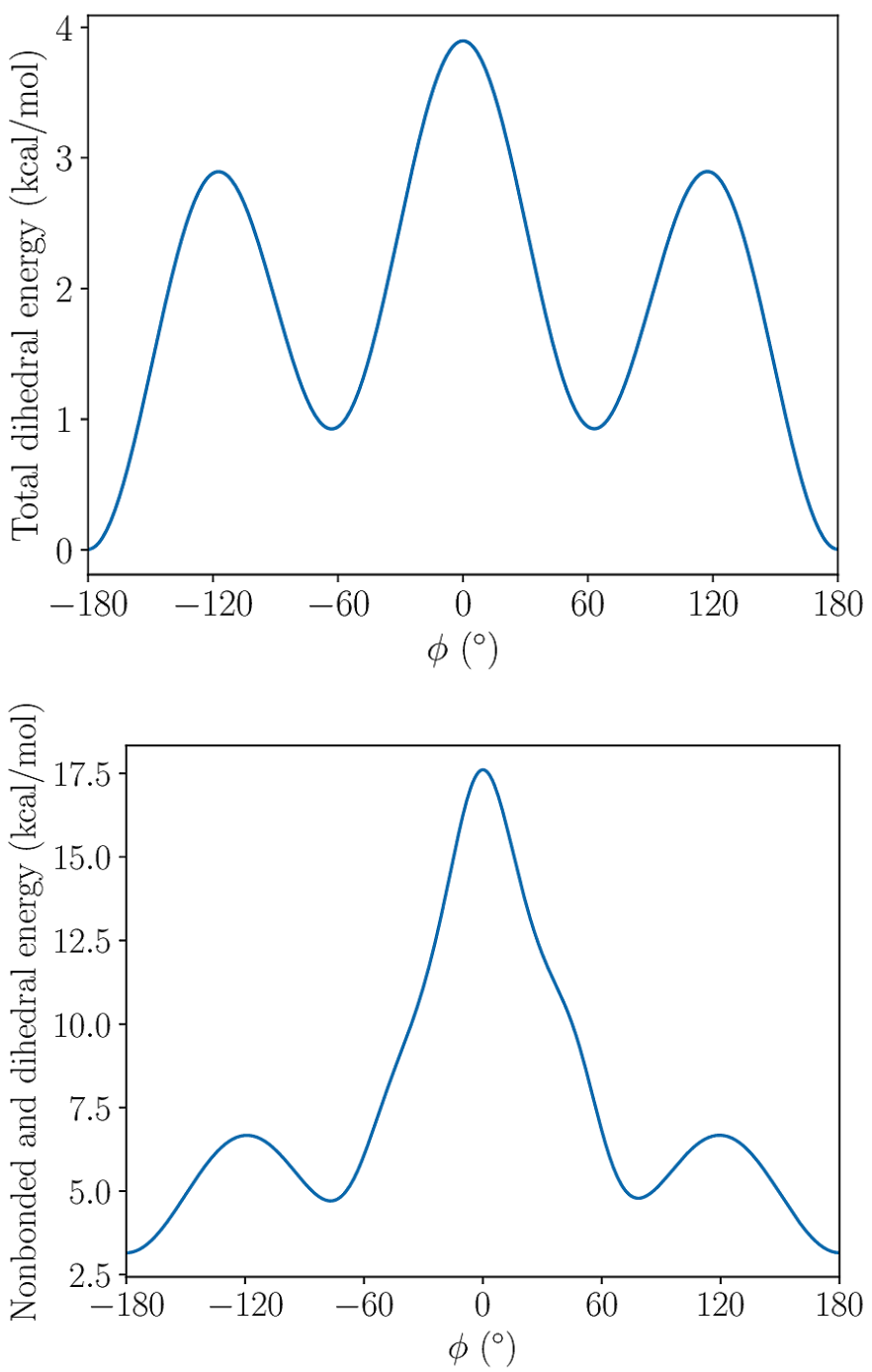

Figure S4: The energy profile of the OPLS-AA force field for the n-octane rotating around the C9-C12 bond with two rigid parts: (top) only the torsional angle and (bottom) the total energy including the bonded and non-bonded terms of the potential. In the torsional energy a global minimum is observed in $\pm 180^{\circ}$, trans form, and another two minima is observed in $\pm 60^{\circ}$, gauche $( \pm)$ forms, with $\Delta E$ (trans $\rightarrow$ gauche $)=0.9 \mathrm{kcal} / \mathrm{mol}$ and barriers of $\Delta E^{*}($ trans $\rightarrow$ gauche $)=2.9 \mathrm{kcal} / \mathrm{mol}$ and $\Delta E^{*}($ gauche $\rightarrow$ gauche $)=3.9 \mathrm{kcal} / \mathrm{mol}$. Considering only this torsional energy, the $\Delta E($ trans $\rightarrow$ gauche $)=0.9 \mathrm{kcal} / \mathrm{mol}$ gives a trans:gauche population ratio of 70:30. In the total energy the global minimum still in trans form and the other two minima in gauche $( \pm)$ forms shifted to around $\pm 75^{\circ} \mathrm{w}$ ith $\Delta E($ trans $\rightarrow$ gauche $)=1.6 \mathrm{kcal} / \mathrm{mol}$ and barriers of $\Delta E^{*}($ trans $\rightarrow$ gauche $)=3.5 \mathrm{kcal} / \mathrm{mol}$ and $\Delta E^{*}($ gauche $\rightarrow$ gauche $)=12.8 \mathrm{kcal} / \mathrm{mol}$. Considering the total rotational energy, the $\Delta E($ trans $\rightarrow$ gauche $)=1.6 \mathrm{kcal} / \mathrm{mol}$ gives a trans:gauche population ratio of 88:12. We considered these populations as theoretical ideal values because they were obtained with rigid n-octane rotation not including the angles relaxation that were considered in our simulations. 


\section{SI 2.3: Simulations analysis}
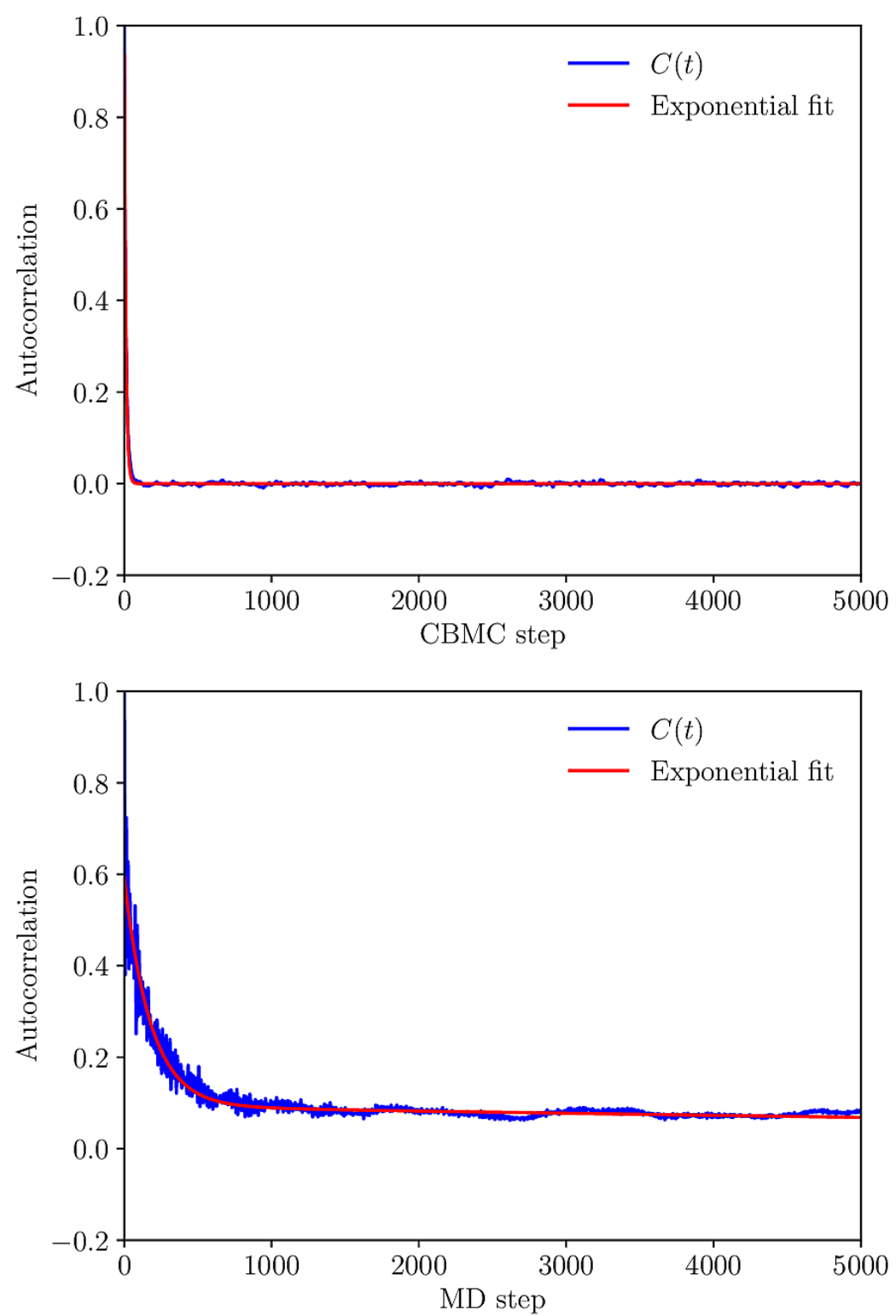

Figure S5: The autocorrelation function for the energy of the isolated n-octane simulations with CBMC (top) and MD (bottom). The fitted functions were two exponential decay, $C(t)=w_{1} e^{-t / \tau_{1}}+w_{2} e^{-t / \tau_{2}}$ where $w_{1}=9.33 \times 10^{-1}, \tau_{1}=1.11 \times 10^{1}, w_{2}=2.50 \times 10^{-4}$ and $\tau_{2}=9.44 \times 10^{4}$ (top) and $w_{1}=4.99 \times 10^{-1}, \tau_{1}=1.78 \times 10^{2}, w_{2}=9.28 \times 10^{-2}$ and $\tau_{2}=1.64 \times 10^{4}$ (bottom). The statistical correlation interval (or time) was obtained as $s=2 \tau$, with $\tau=w_{1} \tau_{1}+w_{2} \tau_{2}$ by the integration of $C(t)$. Then, $s_{\mathrm{CBMC}}=68$ and $s_{\mathrm{MD}}=3225$. Therefore, the CBMC method generate statistically uncorrelated configurations faster than MD method, in this case around 50 times. 
Table S2: Average and standard deviation of the angles from the sampled n-octane configurations in CBMC and MD simulations of the isolated molecule and in chloroform solution. All values are in ${ }^{\circ}$ and they show a very good agreement between both simulations, as expected.

\begin{tabular}{lllll}
\hline & \multicolumn{2}{c}{ Isolated } & \multicolumn{2}{c}{ In chloroform } \\
\cline { 2 - 5 } & CBMC & MD & CBMC & MD \\
\hline H-CH3-CH2 & $111.6 \pm 4.4$ & $111.4 \pm 4.5$ & $111.2 \pm 4.6$ & $111.4 \pm 4.6$ \\
H-CH3-H & $107.6 \pm 4.8$ & $107.6 \pm 4.9$ & $107.6 \pm 4.7$ & $107.6 \pm 4.9$ \\
CH3-CH2-CH2 & $113.1 \pm 3.4$ & $112.7 \pm 3.5$ & $113.1 \pm 3.4$ & $112.7 \pm 3.4$ \\
H-CH2-CH2 & $110.2 \pm 4.3$ & $110.0 \pm 4.4$ & $109.8 \pm 4.4$ & $110.0 \pm 4.5$ \\
H-CH2-H & $106.2 \pm 4.7$ & $106.5 \pm 4.8$ & $106.3 \pm 4.8$ & $106.5 \pm 4.8$ \\
CH2-CH2-CH2 & $113.1 \pm 3.4$ & $112.8 \pm 3.5$ & $113.0 \pm 3.4$ & $112.8 \pm 3.5$ \\
\hline
\end{tabular}
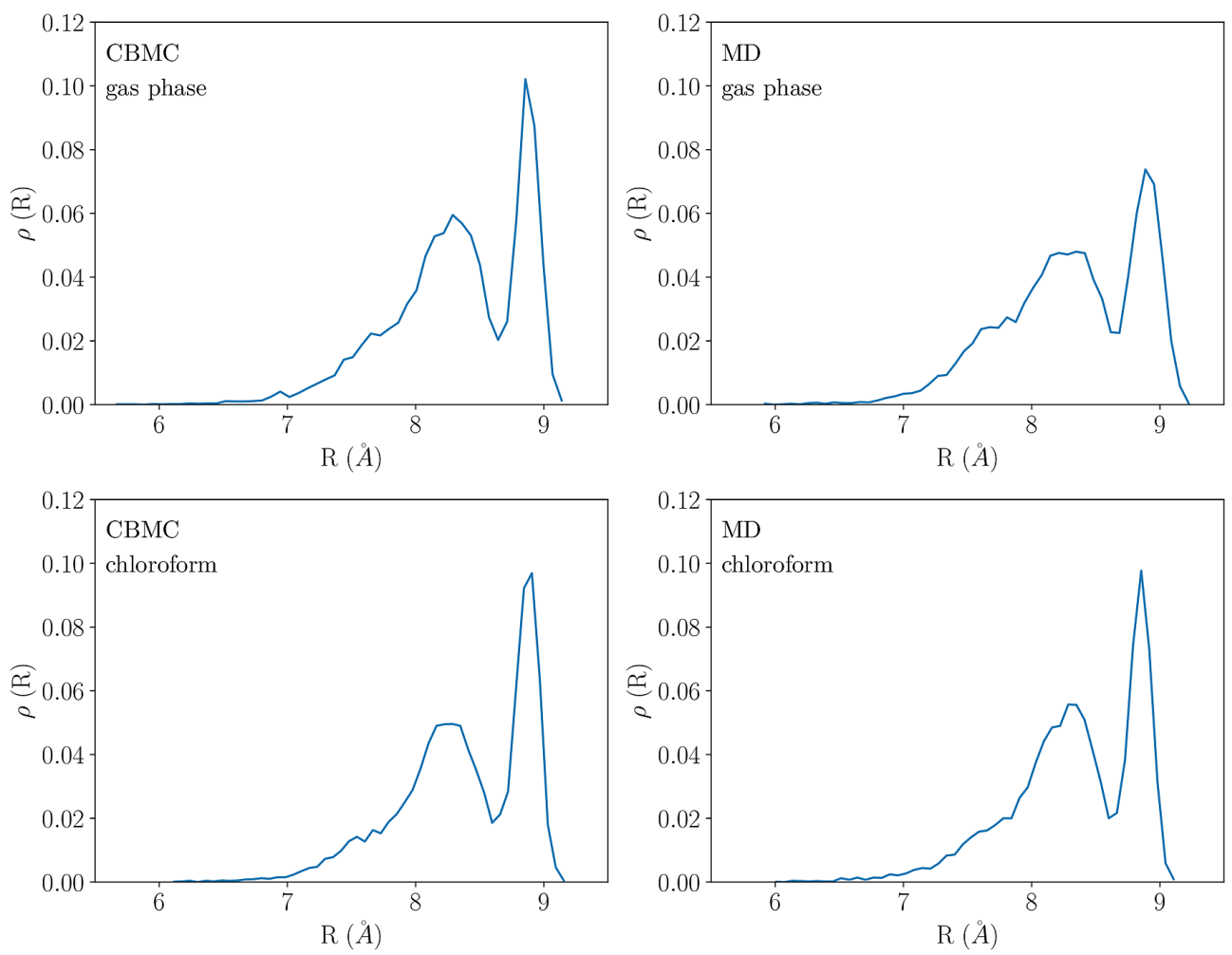

Figure S6: Calculated distribution of end-to-end lengths $R$ of sampled n-octane configurations in $\mathrm{CBMC}$ and MD simulations of the isolated molecule (gas phase) and in chloroform solution. The well defined peak at larger lengths (between $8.7 \AA$ and $9.1 \AA$ ) describes the all-trans conformation and the broad and asymmetrical peak at low lengths (between $6.5 \AA$ and $8.7 \AA$ ) describes the other conformations mixing the trans and gauche forms of the five dihedral angles of n-octane. 


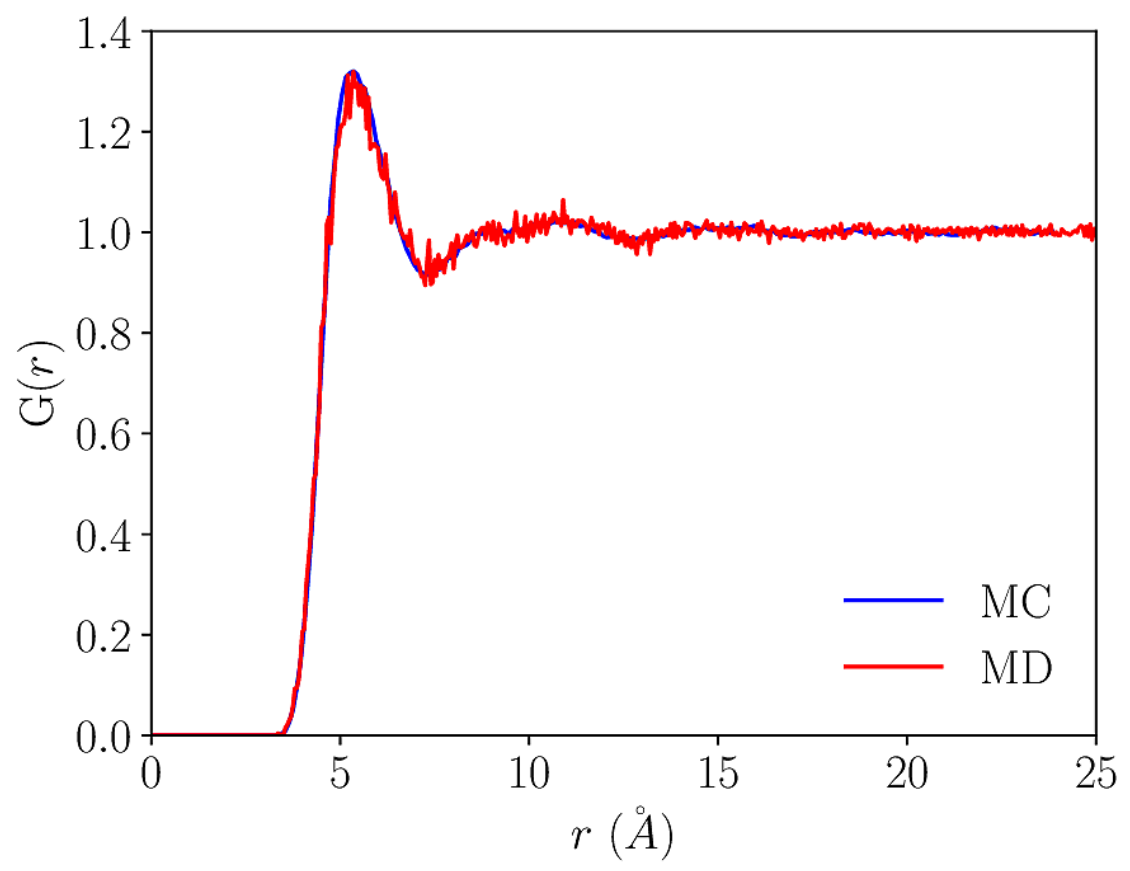

Figure S7: Radial distribution function $G(r)$ between the center of mass of octane and the center of mass of the chloroform molecules, calculated using the CBMC simulation (blue) with $10^{5}$ configurations and MD simulation (red) with with $10^{4}$ configurations. As one can see, the agreement between the curves show that the solvation shells are the same, independent of the method used for the simulation. 

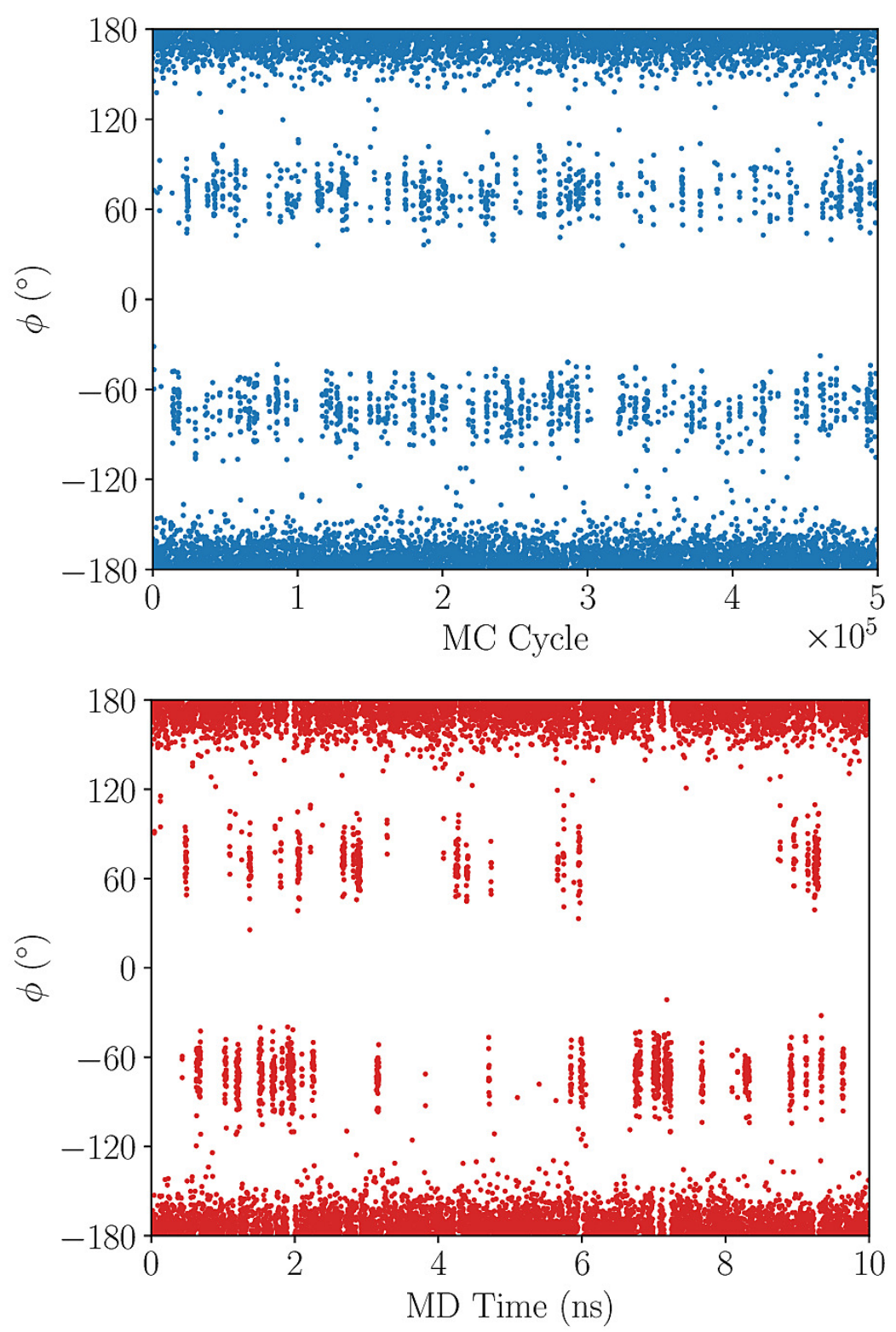

Figure S8: Evolution of the dihedral angle C6-C9-C12-C15 of n-octane in chloroform with simulation steps. Similar to the isolated molecule, the gauche $(-)$, gauche $(+)$ and trans forms can be easily identified and in the CBMC simulation the frequency of barrier crossing is still larger than that obtained in the MD simulation. The vertical strips in the MD distribution are more defined than in CBMC distribution and it describes the time interval where the conformation is trapped in the energy minimum. Additionally, in the MD distribution an asymmetry shows up between the gauche $(-)$ and gauche $(+)$ forms, which the gauche $(-)$ has a larger occurrence than gauche $(+)$ form, indicating the need of a larger MD simulation to improve the statistics of the frequency of barrier crossing. 


\section{SI 3: DCE}
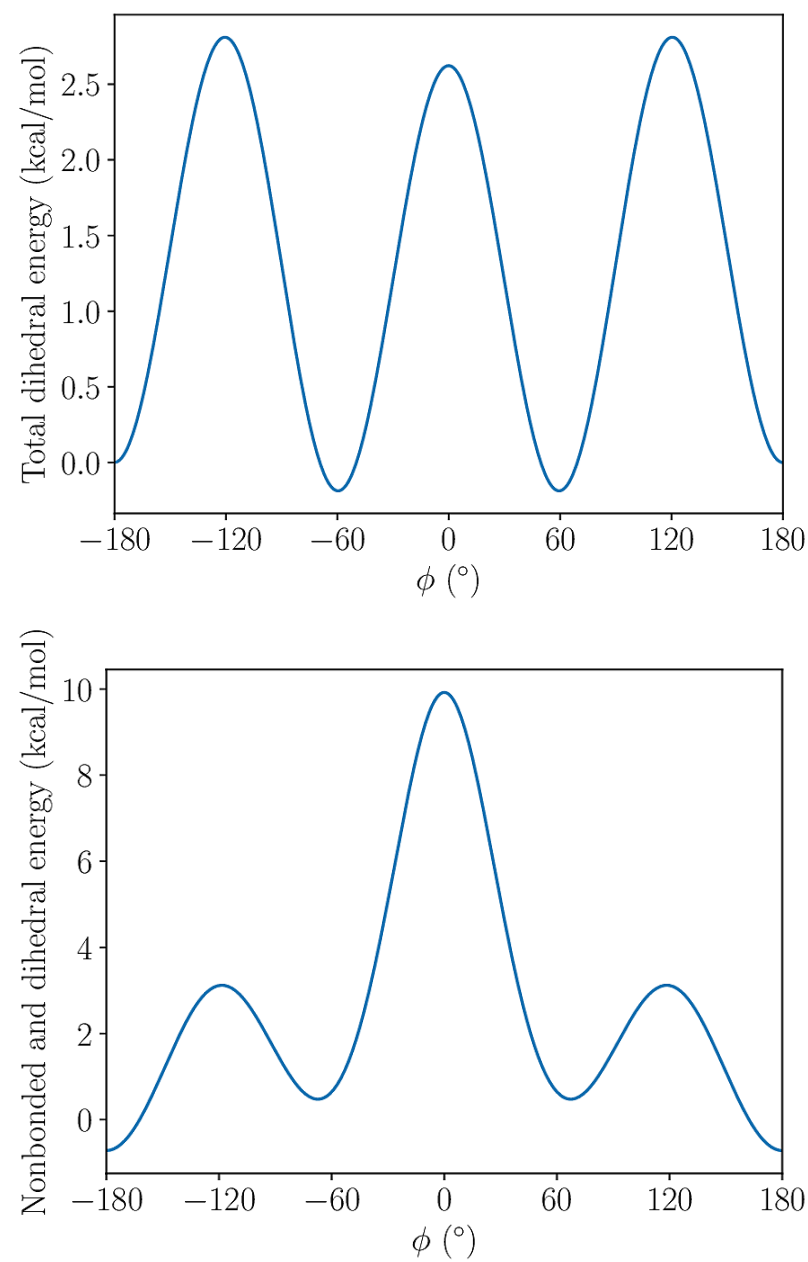

Figure S9: The energy profile of the OPLS-AA force field for the DCE rotating around the $\mathrm{C}-\mathrm{C}$ bond with two rigid parts: (top) only the torsional angle and (bottom) the total energy including the bonded and non-bonded terms of the potential. In the torsional energy a global minima are observed around $\pm 60^{\circ}$, gauche form, and another minimum is observed in $\pm 180^{\circ}$, trans forms, with $\Delta E$ (trans $\rightarrow$ gauche $)=-0.2 \mathrm{kcal} / \mathrm{mol}$ and barriers of $\Delta E^{*}($ trans $\rightarrow$ gauche $)=2.8 \mathrm{kcal} / \mathrm{mol}$ and $\Delta E^{*}($ gauche $\rightarrow$ gauche $)=2.6 \mathrm{kcal} / \mathrm{mol}$. Considering only this torsional energy, the $\Delta E($ trans $\rightarrow$ gauche $)=-0.2 \mathrm{kcal} / \mathrm{mol}$ gives a trans:gauche population ratio of 26:73. In the total energy the global minimum now in trans form and the other two minima in gauche $( \pm)$ forms shifted to around $\pm 70^{\circ}$ with $\Delta E($ trans $\rightarrow$ gauche $)=1.2 \mathrm{kcal} / \mathrm{mol}$ and barriers of $\Delta E^{*}($ trans $\rightarrow$ gauche $)=3.8 \mathrm{kcal} / \mathrm{mol}$ and $\Delta E^{*}($ gauche $\rightarrow$ gauche $)=9.4 \mathrm{kcal} / \mathrm{mol}$. Considering the total rotational energy, the $\Delta E($ trans $\rightarrow$ gauche $)=1.2 \mathrm{kcal} / \mathrm{mol}$ gives a trans:gauche population ratio of 80:20. We considered these populations as theoretical ideal values because they were obtained with rigid DCE rotation not including the bonds and angles relaxation that were considered in our simulations. 

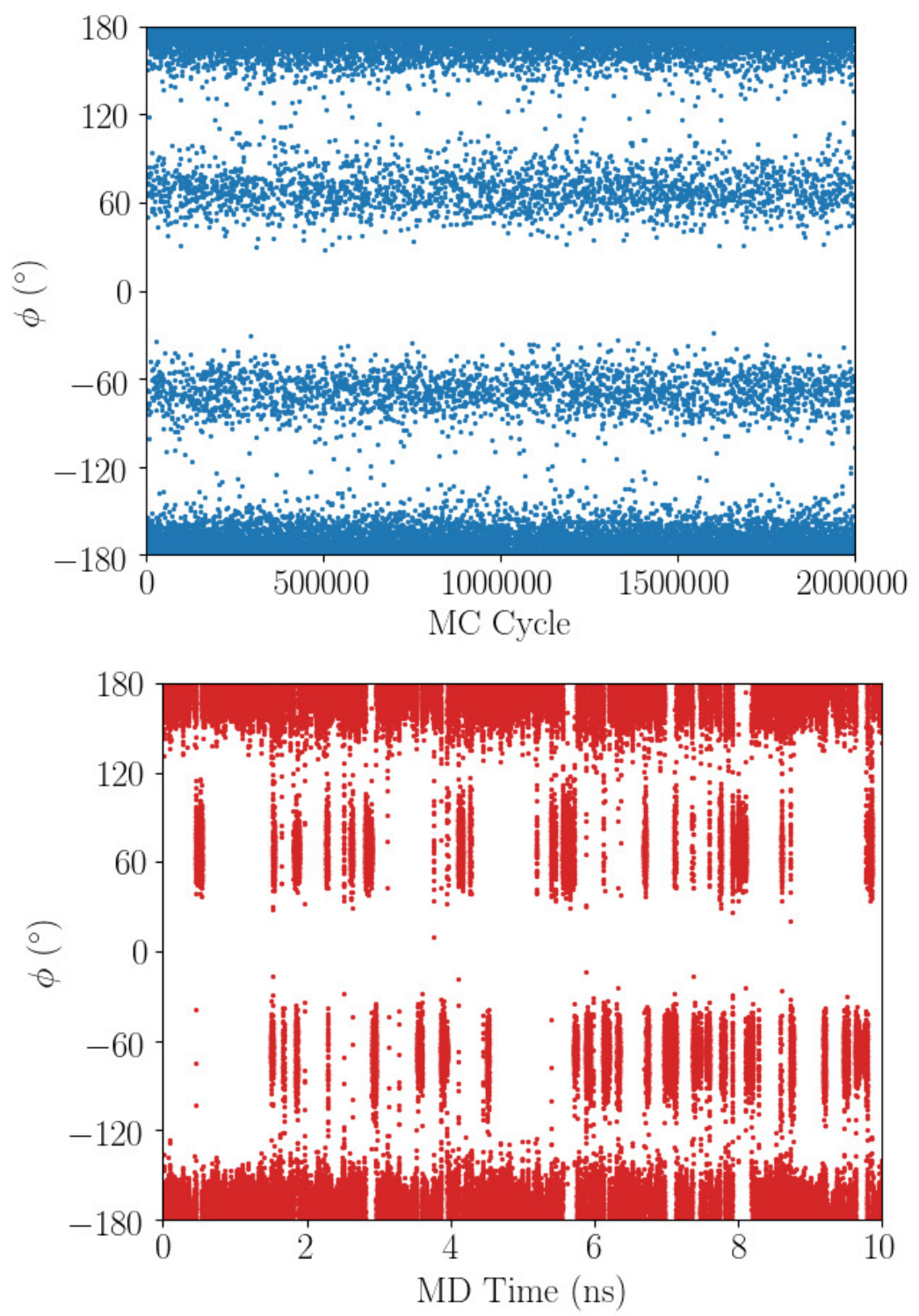

Figure S10: Evolution of the dihedral angle Cl-C-C-Cl of isolated DCE with simulation steps. The gauche $(-)$, gauche $(+)$ and trans forms can be easily identified with a larger density at the trans form. In the CBMC simulation the distribution is more uniform showing a larger frequency of barrier crossing than that obtained in the MD simulation. The vertical strips in the MD distribution are well defined and show the time interval where the conformation is trapped in the energy minimum. 

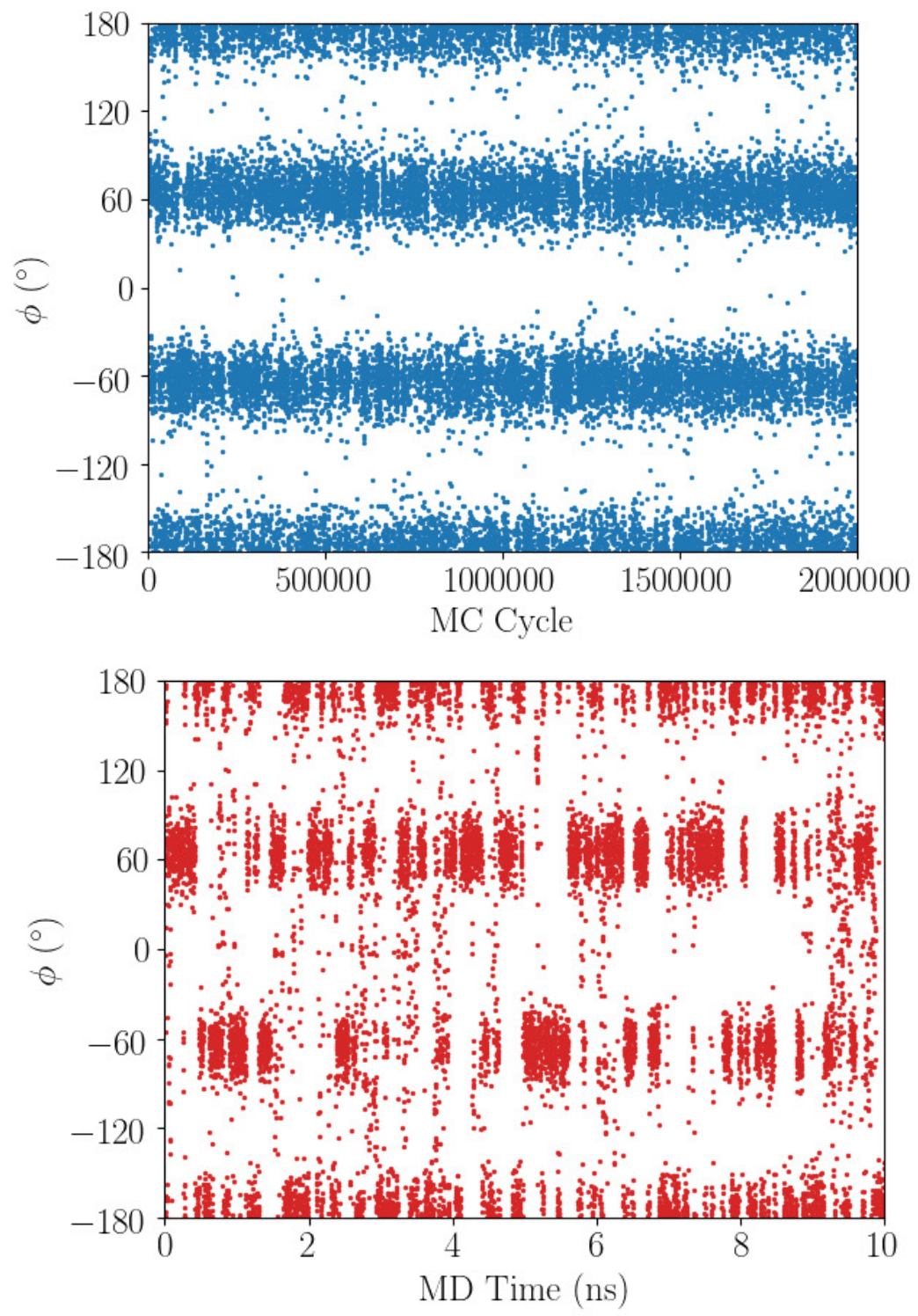

Figure S11: Evolution of the dihedral angle Cl-C-C-Cl of liquid DCE with simulation steps. Comparatively with the isolated DCE (see Fig. S10), it can be notice a increasing in the gauche form. 


\section{SI 4: Data from the SubPC simulations}

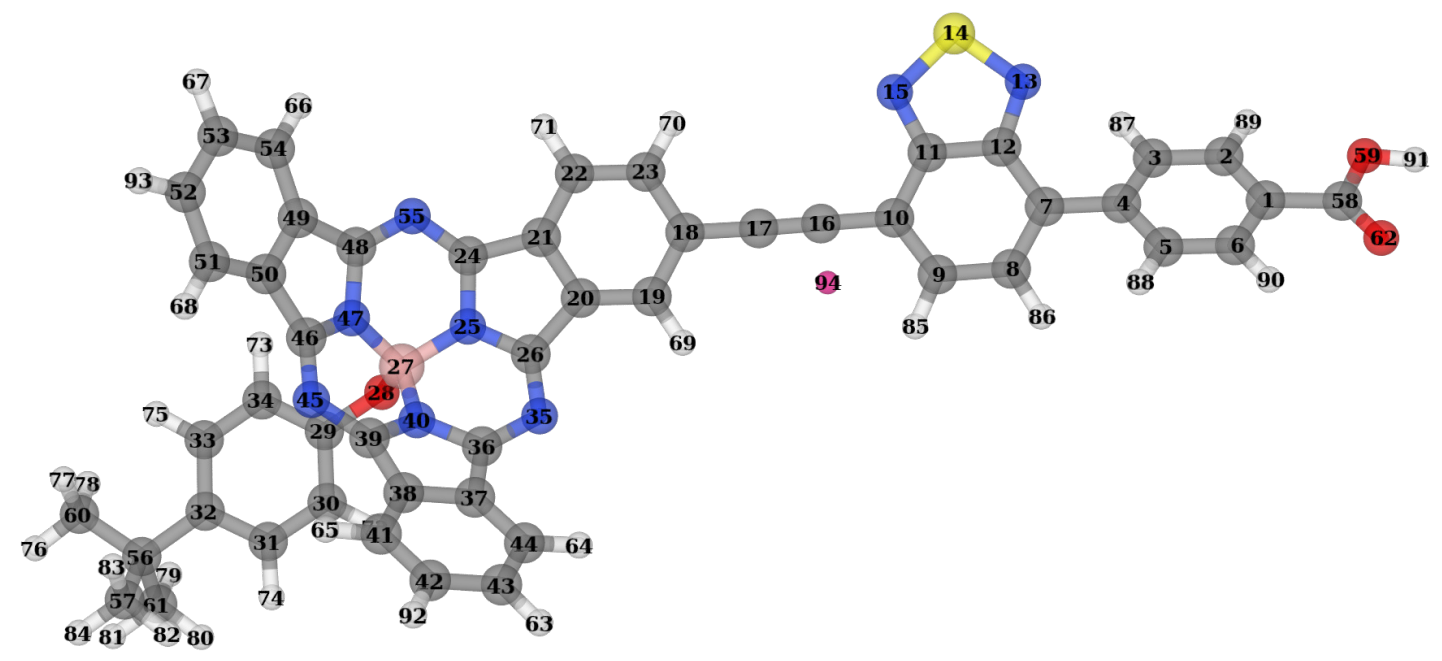

Figure S12: SubPC molecule with numeric labels for the atoms. 

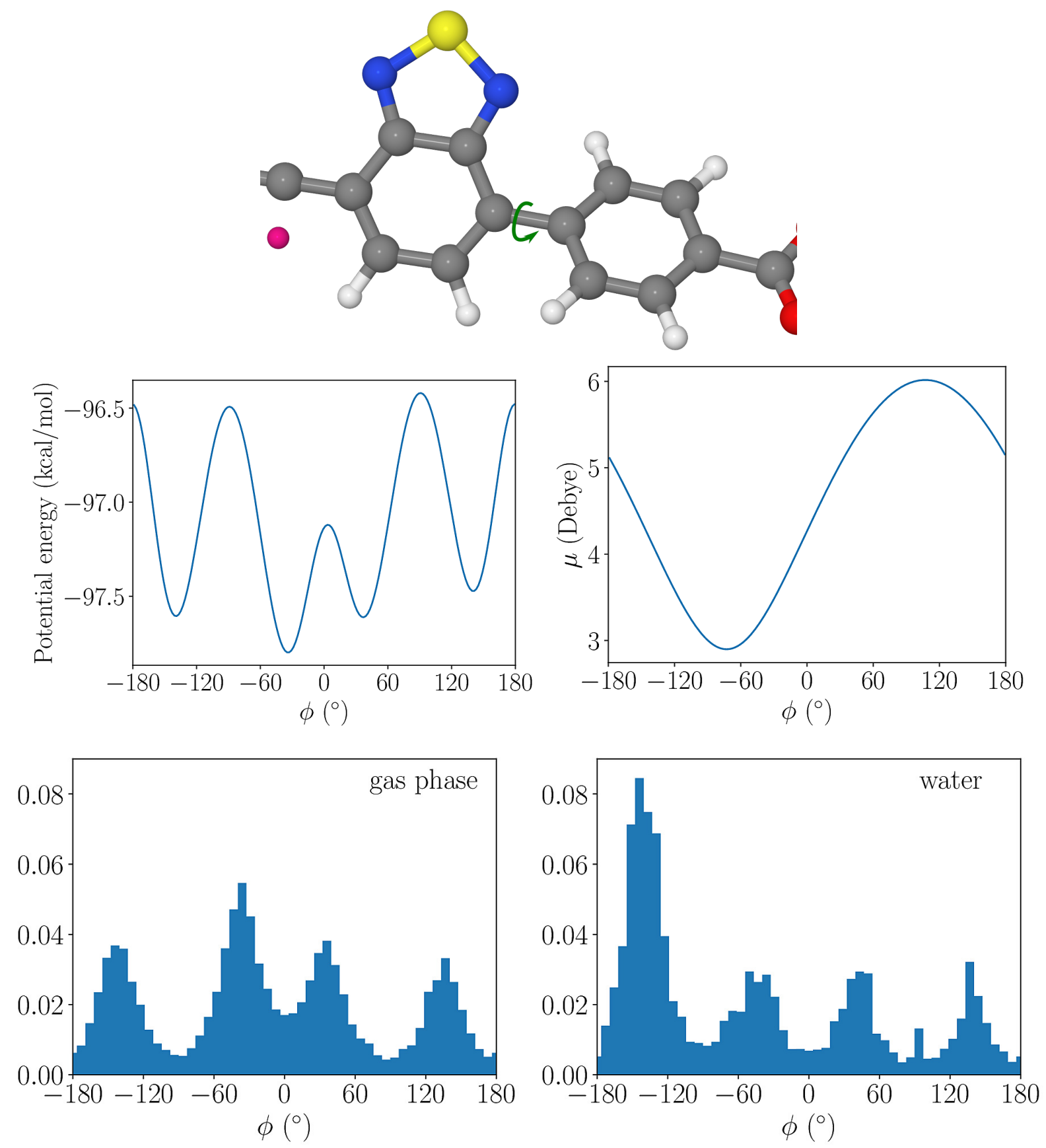

Figure S13: Top: Illustration the dihedral rotation $D 1$ between fragments 1 and 2; Middle left: The total energy profile involved in this rotation (C5-C4-C7-C8) for the isolated SubPC; Middle Right: Dipole moment $\mu$ as a function of the rotation; Bottom left: Dihedral distribution obtained with the CBMC simulation of the isolated SubPC (gas phase); and Bottom right: Distribution obtained with the CBMC simulation of SubPC in water. 

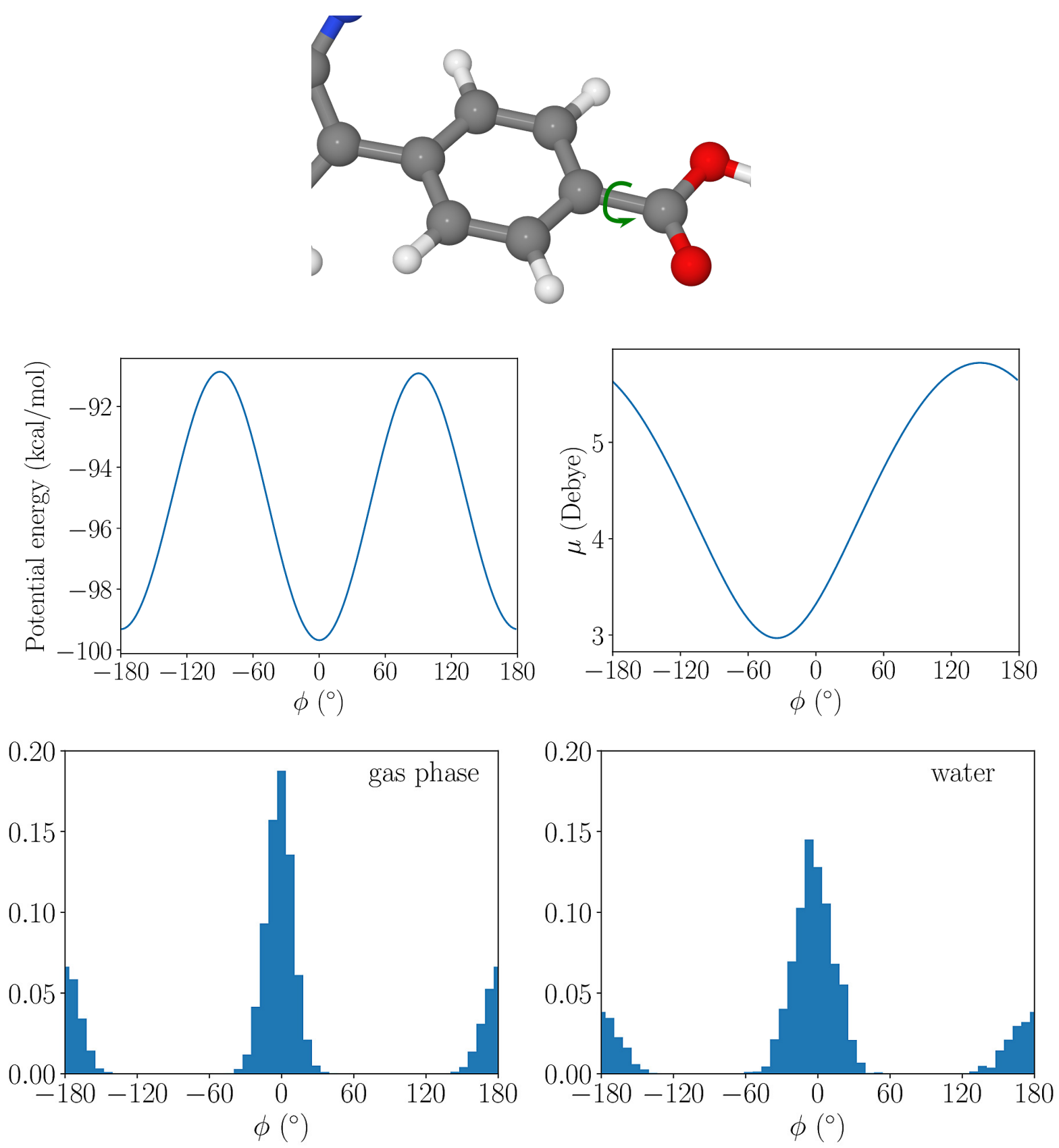

Figure S14: Top: Illustration the dihedral rotation $D 2$ between fragments 1 and 8; Middle left: The total energy profile involved in this rotation (C6-C1-C58-O62) for the isolated SubPC; Middle Right: Dipole moment $\mu$ as a function of the rotation; Bottom left: Dihedral distribution obtained with the CBMC simulation of the isolated SubPC (gas phase); and Bottom right: Distribution obtained with the CBMC simulation of SubPC in water. 

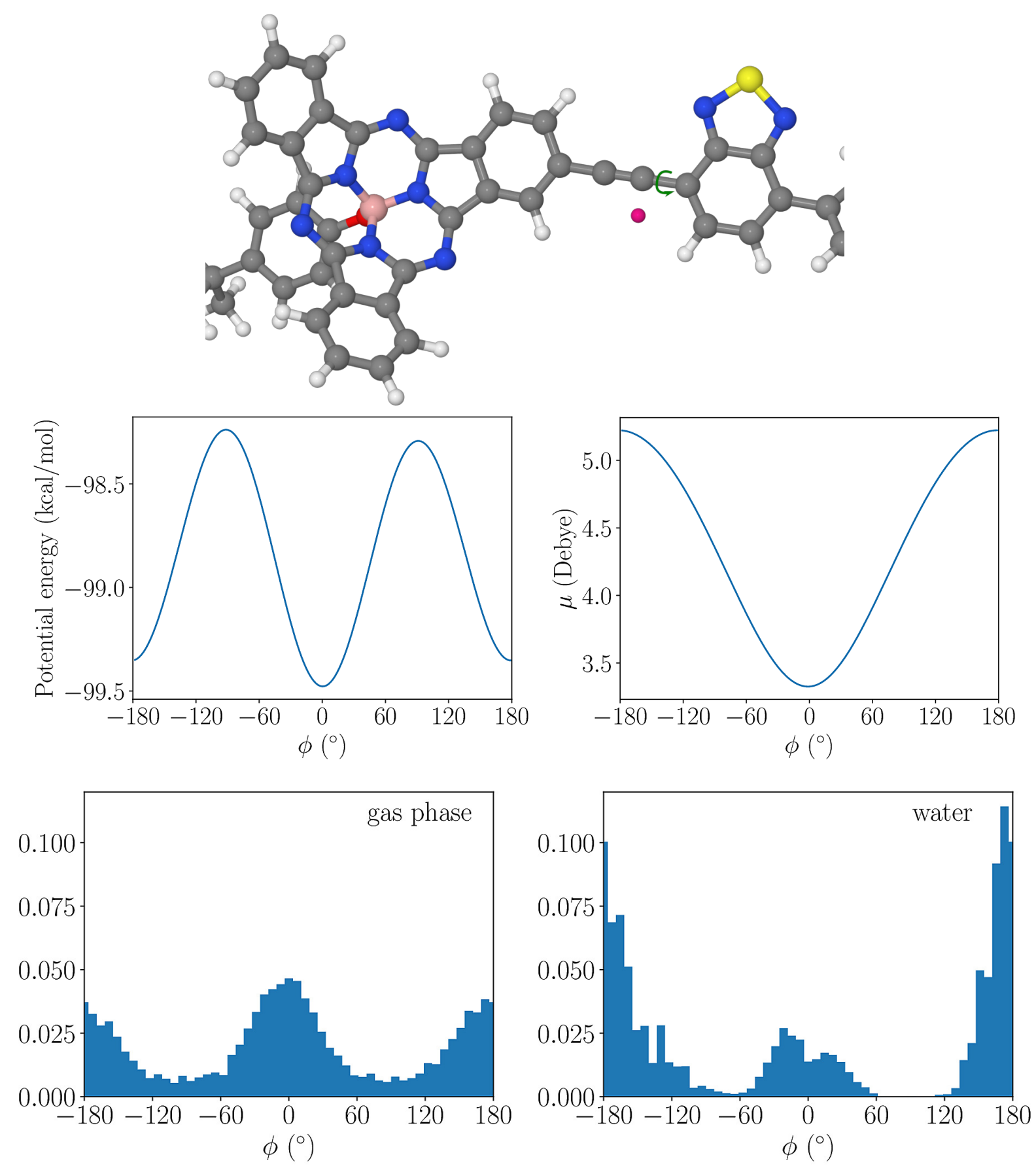

Figure S15: Top: Illustration the dihedral rotation D3 between fragments 2 and 3; Middle left: The total energy profile involved in this rotation (C9-C10-C16-Xx94) for the isolated SubPC; Middle Right: Dipole moment $\mu$ as a function of the rotation; Bottom left: Dihedral distribution obtained with the CBMC simulation of the isolated SubPC (gas phase); and Bottom right: Distribution obtained with the CBMC simulation of SubPC in water. 

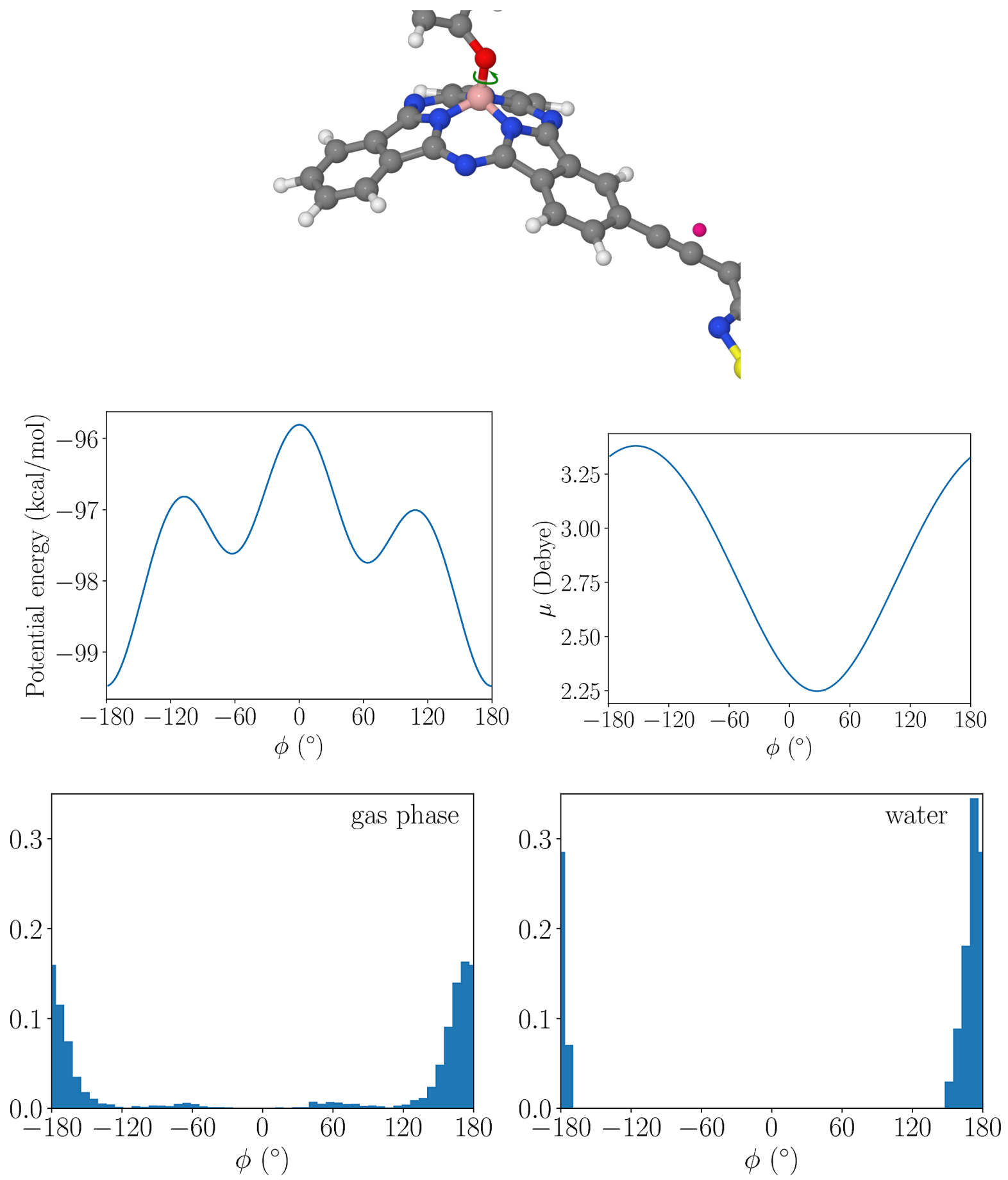

Figure S16: Top: Illustration the dihedral rotation $D 4$ between fragments 3 and 4; Middle left: The total energy profile involved in this rotation (N25-B27-O28-C29) for the isolated SubPC; Middle Right: Dipole moment $\mu$ as a function of the rotation; Bottom left: Dihedral distribution obtained with the CBMC simulation of the isolated SubPC (gas phase); and Bottom right: Distribution obtained with the CBMC simulation of SubPC in water. 


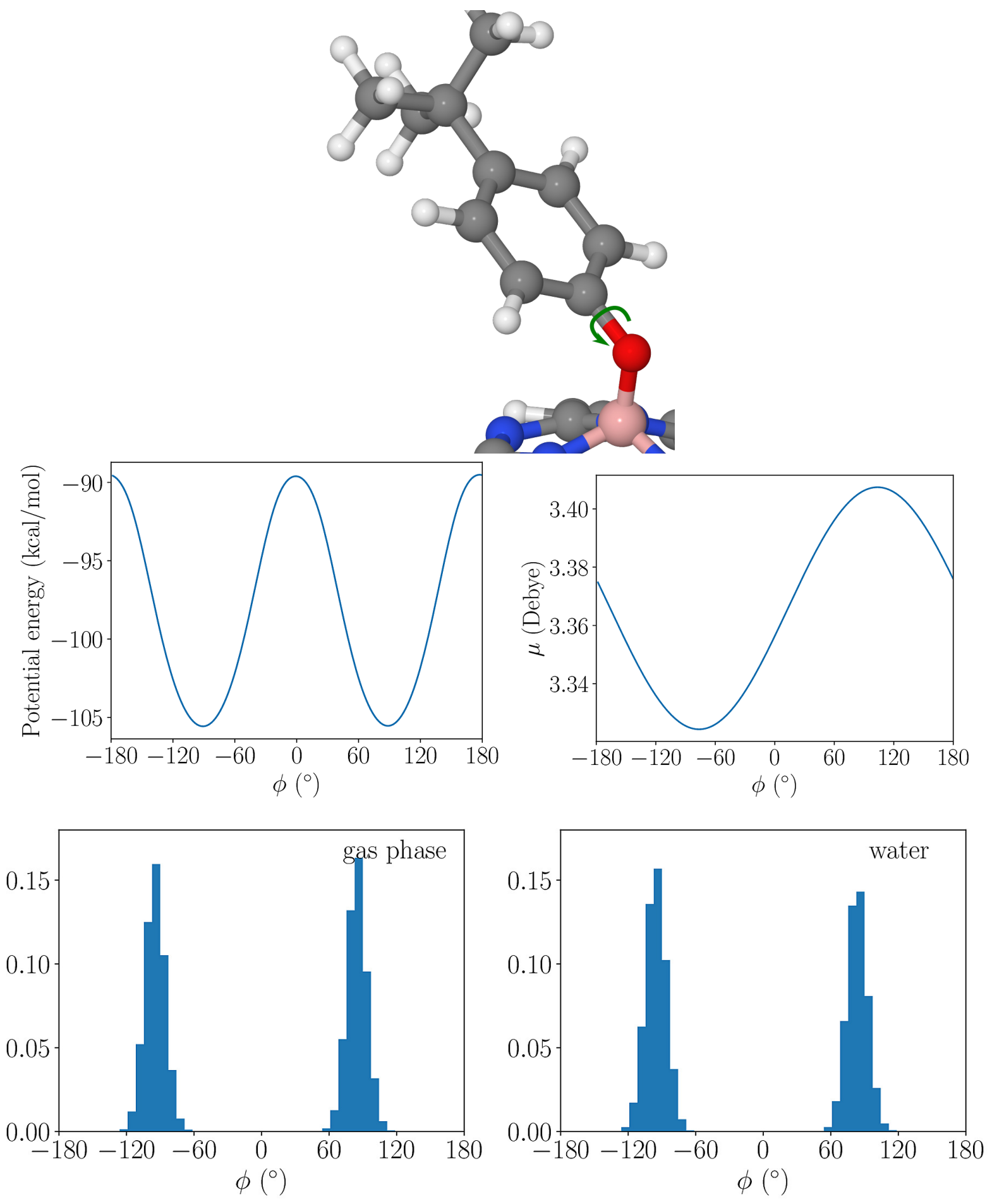

Figure S17: Top: Illustration the dihedral rotation $D 5$ between fragments 4 and 5; Middle left: The total energy profile involved in this rotation (B27-O28-C29-C30) for the isolated SubPC; Middle Right: Dipole moment $\mu$ as a function of the rotation; Bottom left: Dihedral distribution obtained with the CBMC simulation of the isolated SubPC (gas phase); and Bottom right: Distribution obtained with the CBMC simulation of SubPC in water. 

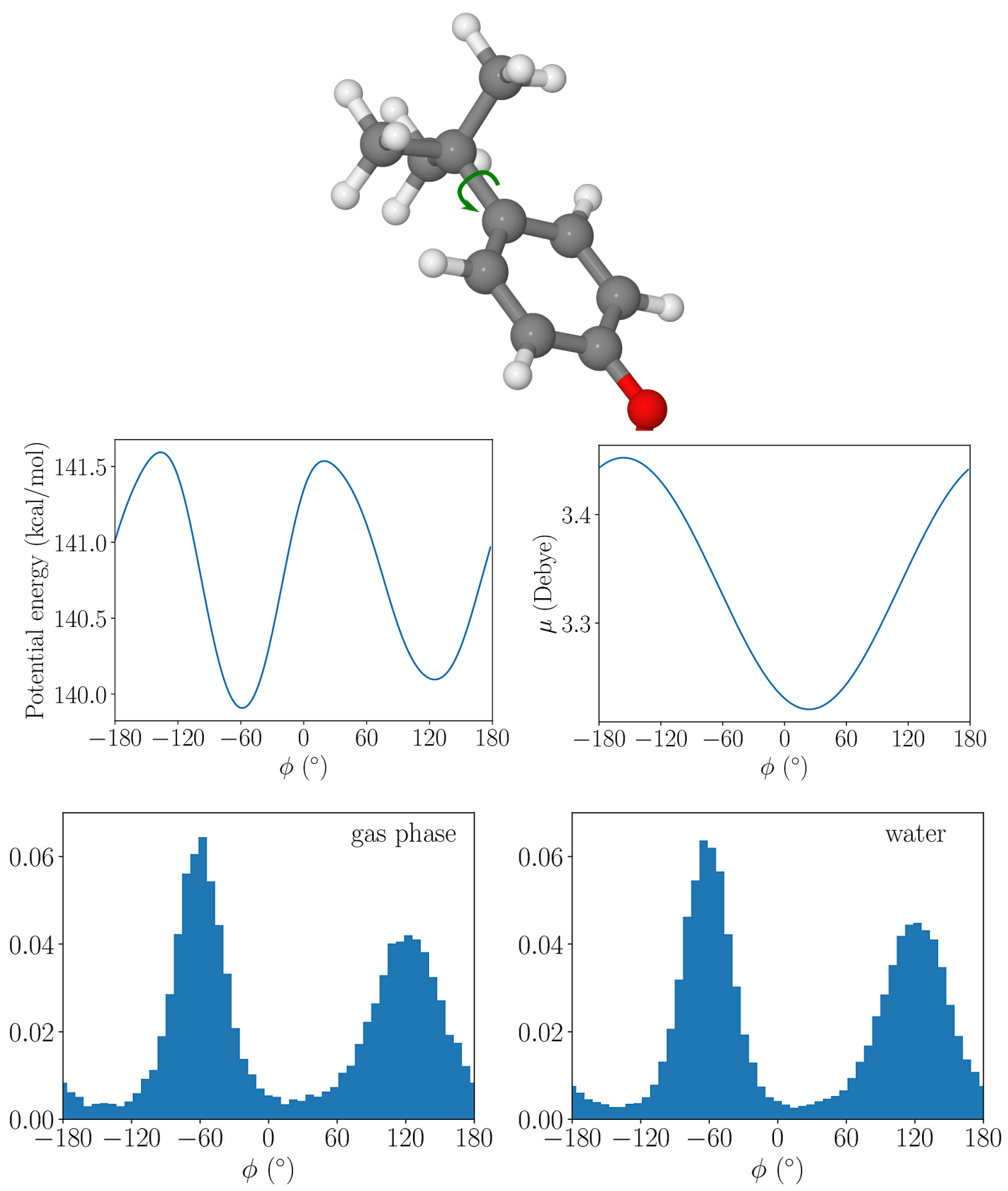

Figure S18: Top: Illustration the dihedral rotation $D 6$ between fragments 5 and 6; Middle left: The total energy profile involved in this rotation (C31-C32-C56-C61) for the isolated SubPC; Middle Right: Dipole moment $\mu$ as a function of the rotation; Bottom left: Dihedral distribution obtained with the CBMC simulation of the isolated SubPC (gas phase); and Bottom right: Distribution obtained with the CBMC simulation of SubPC in water. 

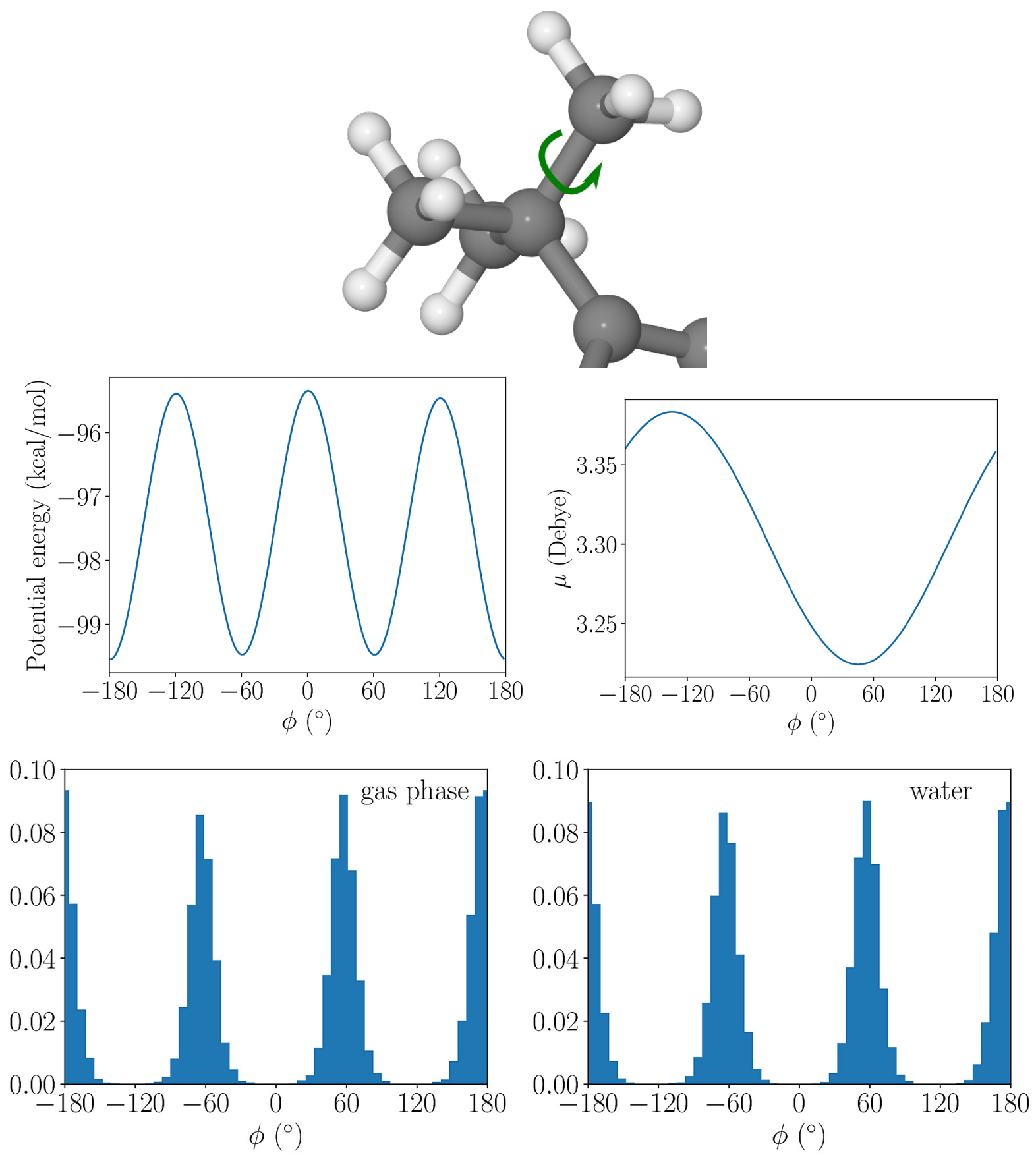

Figure S19: Top: Illustration the dihedral rotation $D 7$ between fragments 6 and 7; Middle left: The total energy profile involved in this rotation (C32-C56-C57-H82) for the isolated SubPC; Middle Right: Dipole moment $\mu$ as a function of the rotation; Bottom left: Dihedral distribution obtained with the CBMC simulation of the isolated SubPC (gas phase); and Bottom right: Distribution obtained with the CBMC simulation of SubPC in water. 


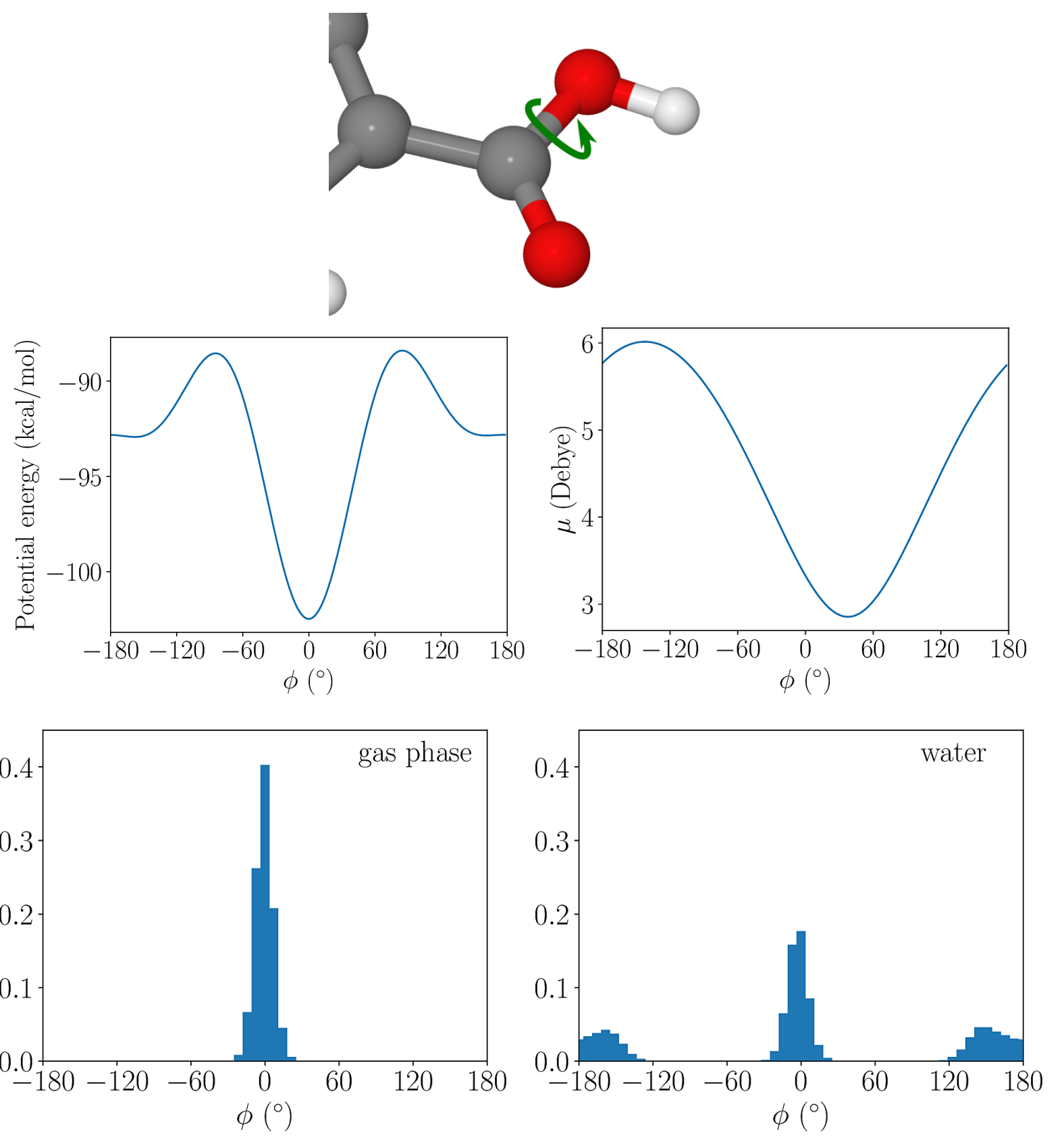

Figure S20: Top: Illustration the dihedral rotation $D 8$ between fragments 8 and 9; Middle left: The total energy profile involved in this rotation (O62-C58-O59-H91) for the isolated SubPC; Middle Right: Dipole moment $\mu$ as a function of the rotation; Bottom left: Dihedral distribution obtained with the CBMC simulation of the isolated SubPC (gas phase); and Bottom right: Distribution obtained with the CBMC simulation of SubPC in water. 


\section{SI 5: Example input files}

Below we provide some example input files, which were used to produce the data reported in this work. The .txt files contain the initial geometry of each type of molecules with Cartesian coordinates of the atoms $\left\{x_{i}, y_{i}, z_{i}\right\}$ in $\AA$, and also the nonbonded parameters: the atomic partial charges $\left\{q_{i}\right\}$ in $e$ and the Lennard-Jones parameters $\left\{\epsilon_{i}\right\}$ and $\left\{\sigma_{i}\right\}$ in $\mathrm{kcal} / \mathrm{mol}$ and $\AA$, respectively. The topology, with the intramolecular potential, is defined in the .dfr file. The input files for the thermalization and CBMC simulation are passed to DICE via shell redirection of input files with the $<$ operator.

These input files were prepared using DICEtools (https ://github.com/hmcezar/dicetools), which provides tools to generate input files from standard chemical structure information and conversion between DICE and GROMACS[12] input files.

\section{SI 5.1: Octane}

Geometry and nonbonded parameters: octane.txt

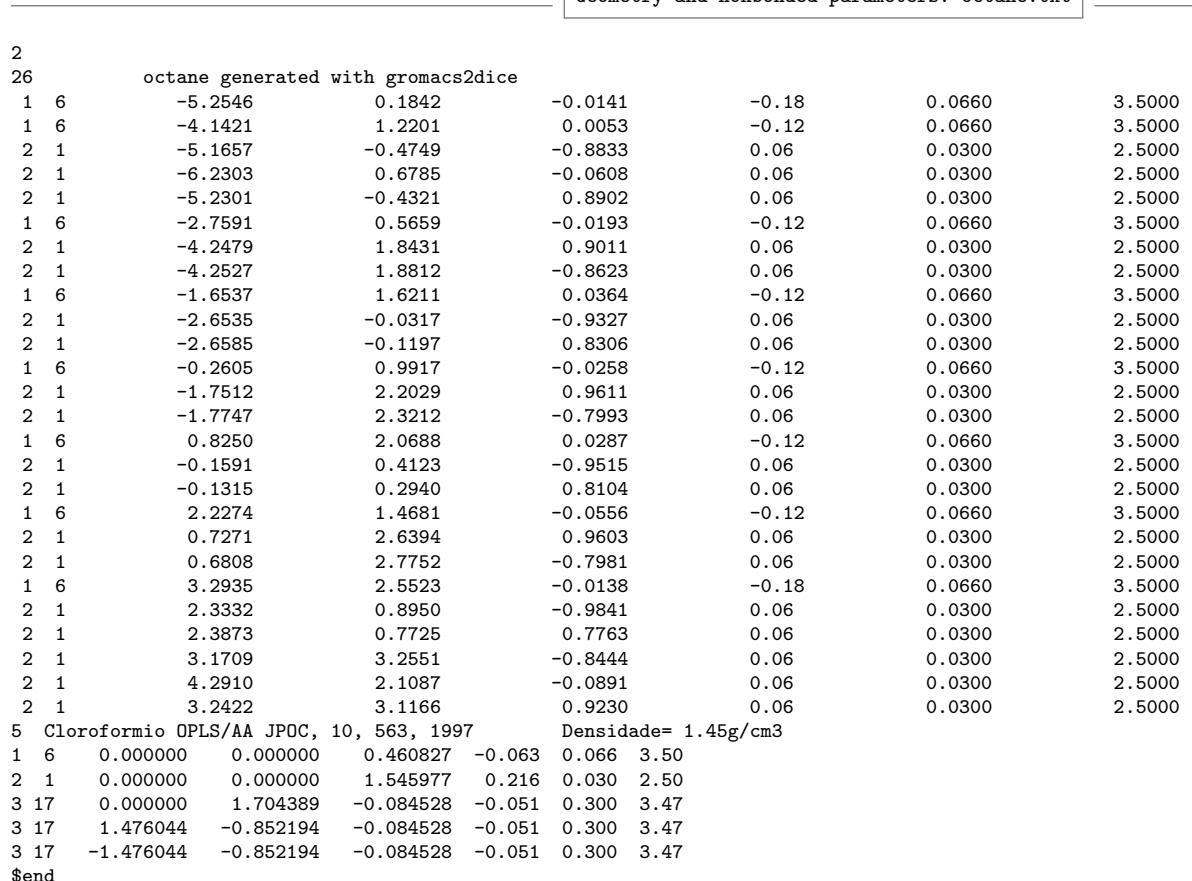

\begin{tabular}{|c|c|c|c|c|c|c|}
\hline & fragments & & & & & \\
\hline 1 & {$[1$} & 2 & 5 & 4 & $3]$ & $\mathrm{F}$ \\
\hline 2 & [ 2 & 6 & 1 & 7 & $8]$ & $\mathrm{F}$ \\
\hline 3 & {$[6$} & 9 & 2 & 11 & $10]$ & F \\
\hline 4 & {$[9$} & 12 & 6 & 13 & $14]$ & $\mathrm{F}$ \\
\hline 5 & [ 12 & 15 & 9 & 17 & $16]$ & $\mathrm{F}$ \\
\hline 6 & [ 15 & 18 & 12 & 19 & $20]$ & $\mathrm{F}$ \\
\hline 7 & [ 18 & 21 & 15 & 23 & $22]$ & $\mathrm{F}$ \\
\hline 8 & [ 21 & 18 & 26 & 25 & $24]$ & $\mathrm{F}$ \\
\hline
\end{tabular}

\$ragment connection

$\begin{array}{ll}1 & 2 \\ 2 & 3 \\ 3 & 4\end{array}$ 
$\begin{array}{ll}4 & 5 \\ 5 & 6 \\ 6 & 7 \\ 7 & 8\end{array}$

\$end fragment connection

\begin{tabular}{|c|c|c|}
\hline \multicolumn{3}{|l|}{ \$bond } \\
\hline 15 & 340.0 & 1.09 \\
\hline 14 & 340.0 & 1.09 \\
\hline 13 & 340.0 & 1.09 \\
\hline 12 & 268.0 & 1.52 \\
\hline 28 & 340.0 & 1.1 \\
\hline 27 & 340.0 & 1.1 \\
\hline 26 & 268.0 & 1.53 \\
\hline 611 & 340.0 & 1.1 \\
\hline 610 & 340.0 & 1.1 \\
\hline 69 & 268.0 & 1.53 \\
\hline $9 \begin{array}{ll}9 & 14\end{array}$ & 340.0 & 1.1 \\
\hline $9 \quad 13$ & 340.0 & 1.1 \\
\hline 912 & 268.0 & 1.53 \\
\hline $12 \quad 17$ & 340.0 & 1.1 \\
\hline 1216 & 340.0 & 1.1 \\
\hline $12 \quad 15$ & 268.0 & 1.53 \\
\hline $15 \quad 20$ & 340.0 & 1.1 \\
\hline $\begin{array}{ll}15 & 19\end{array}$ & 340.0 & 1.1 \\
\hline $\begin{array}{ll}15 & 18\end{array}$ & 268.0 & 1.53 \\
\hline $18 \quad 23$ & 340.0 & 1.1 \\
\hline $18 \quad 22$ & 340.0 & 1.1 \\
\hline 1821 & 268.0 & 1.52 \\
\hline 2126 & 340.0 & 1.1 \\
\hline 2125 & 340.0 & 1.09 \\
\hline 2124 & 340.0 & 1.09 \\
\hline
\end{tabular}

\$angle

$\begin{array}{lll}1 & 2 & 8 \\ 1 & 2 & 7\end{array}$

$\begin{array}{lll}1 & 2 & 7\end{array}$

1256

215

21

$\begin{array}{lll}2 & 1 & 3 \\ 2 & 6 & 11\end{array}$

$\begin{array}{lll}2 & 6 & 11 \\ 2 & 6 & 10\end{array}$

269

315

$\begin{array}{lll}3 & 1 & 4\end{array}$

$4 \begin{array}{lll}4 & 1 & 5\end{array}$

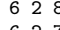

$\begin{array}{lll}6 & 9 & 14 \\ 6 & 9 & 13\end{array}$

$\begin{array}{lll}6 & 9 & 13 \\ 6 & 9 & 12\end{array}$

$\begin{array}{lll}7 & 2 & 8\end{array}$

99611

9610

$\begin{array}{lll}9 & 12 & 17\end{array}$

$9 \begin{array}{lll}9 & 12 & 16\end{array}$

$912 \quad 15$

$\begin{array}{lll}10 & 6 & 11\end{array}$

$\begin{array}{lll}12 & 9 & 14\end{array}$

12913

$\begin{array}{llll}12 & 15 & 20 \\ 12 & 15 & 19\end{array}$

121518

$\begin{array}{lll}13 & 9 & 14\end{array}$

$\begin{array}{lll}15 & 12 & 17\end{array}$

$\begin{array}{lll}15 & 1216\end{array}$

$\begin{array}{lll}15 & 18 & 23\end{array}$

$\begin{array}{lll}15 & 18 & 22\end{array}$

$\begin{array}{lll}15 & 18 & 21\end{array}$

$\begin{array}{lll}16 & 12 & 17\end{array}$

$\begin{array}{lll}18 & 15 & 20\end{array}$

$\begin{array}{lll}18 & 15 & 19\end{array}$

$\begin{array}{lll}18 & 21 & 26 \\ 18 & 21 & 25\end{array}$

$\begin{array}{lll}18 & 21 & 25 \\ 18 & 21 & 24\end{array}$

$\begin{array}{llll}18 & 21 & 24 \\ 19 & 15 & 20\end{array}$

$\begin{array}{lll}21 & 18 & 23\end{array}$

$\begin{array}{lll}21 & 18 & 22\end{array}$

$\begin{array}{lll}22 & 18 & 23\end{array}$

$\begin{array}{lll}24 & 21 & 26\end{array}$

$\begin{array}{llll}24 & 21 & 25 \\ 25 & 21 & 26\end{array}$

\$end angle

harmonic $\quad 37$.

harmonic $\quad 37.5 \quad 109.081$

$\begin{array}{lll} & 37.5 & 109.06\end{array}$

$\begin{array}{llr}\text { harmonic } & 37.5 & 111.727\end{array}$

$\begin{array}{lll}\text { harmonic } & 37.5 & 110.885 \\ \text { harmonic } & 37.5 & 110.147\end{array}$

harmonic $37.5 \quad 110.147$

$\begin{array}{ccc}\text { harmonic } & 37.5 & 111.143 \\ \text { harmonic } & 37.5 & 109.778\end{array}$

$\begin{array}{lll}\text { harmonic } & 37.5 & 109.421\end{array}$

$\begin{array}{lll}\text { harmonic } & 58.35 & 110.966\end{array}$

$\begin{array}{lll}\text { harmonic } & 33.0 & 108.373\end{array}$

harmonic $\quad 33.0 \quad 108.103$

$\begin{array}{lll}\text { harmonic } & 33.0 & 108.086\end{array}$

$\begin{array}{lll}\text { harmonic } & 37.5 & 109.692\end{array}$

$\begin{array}{lll}\text { harmonic } & 37.5 & 110.065\end{array}$

$\begin{array}{lrr}\text { harmonic } & 37.5 & 109.479\end{array}$

$\begin{array}{llc}\text { harmonic } & 37.5 & 109.421 \\ \text { harmonic } & 58.35 & 111.916\end{array}$

$\begin{array}{lll}\text { harmonic } & 33.0 & 107.098\end{array}$

$\begin{array}{lll}\text { harmonic } & 37.5 & 109.703\end{array}$

$\begin{array}{lll}\text { harmonic } & 37.5 & 109.688\end{array}$

$\begin{array}{lll}\text { harmonic } & 37.5 & 109.743\end{array}$

$\begin{array}{lll} & 37.5 & 109.6\end{array}$

harmonic $\quad 58.35 \quad 110.805$

$\begin{array}{lll}\text { harmonic } & 33.0 & 107.207\end{array}$

$\begin{array}{lll}\text { harmonic } & 37.5 & 109.394\end{array}$

$\begin{array}{lll}\text { harmonic } & 37.5 & 109.431\end{array}$

$\begin{array}{lll}\text { harmonic } & 37.5 & 109.447 \\ \text { harmonic } & 37.5 & 109.467\end{array}$

$\begin{array}{lll}\text { harmonic } & 37.35 & 111.834\end{array}$

harmonic 111.83

$\begin{array}{lll}\text { harmonic } & 33.0 & 107.078\end{array}$

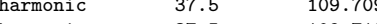

$\begin{array}{lll}\text { harmonic } & 37.5 & 109.719 \\ \text { harmonic } & 37.5 & 109.962\end{array}$

$\begin{array}{lll}\text { harmonic } & 37.5 & 109.974\end{array}$

$\begin{array}{lll}\text { harmonic } & 58.35 & 111.206\end{array}$

$\begin{array}{lll}\text { harmonic } & 33.0 & 107.192\end{array}$

$\begin{array}{lll}\text { harmonic } & 37.5 & 109.374\end{array}$

harmonic $\quad 37.5 \quad 109.506$

harmonic

harmonic 37.5

$\begin{array}{lll}\text { harmonic } & 37.5 & 110.965\end{array}$

harmonic $\quad 33.0 \quad 107.096$

$\begin{array}{lll}\text { harmonic } & 37.5 & 109.204\end{array}$

$\begin{array}{lll}\text { harmonic } & 37.5 & 109.151\end{array}$

$\begin{array}{lll}\text { harmonic } & 33.0 & 107.252\end{array}$

$\begin{array}{lll}\text { harmonic } & 33.0 & 108.18\end{array}$

harmonic $33.0 \quad 108.096$

$\begin{array}{lll}\text { harmonic } & 33.0 & 108.113\end{array}$

\$dihedral

$\begin{array}{llll}\text { OPLS } & -0.0 & -0.0 & 0.3\end{array}$

0.0

0.0

0.0

0.0 


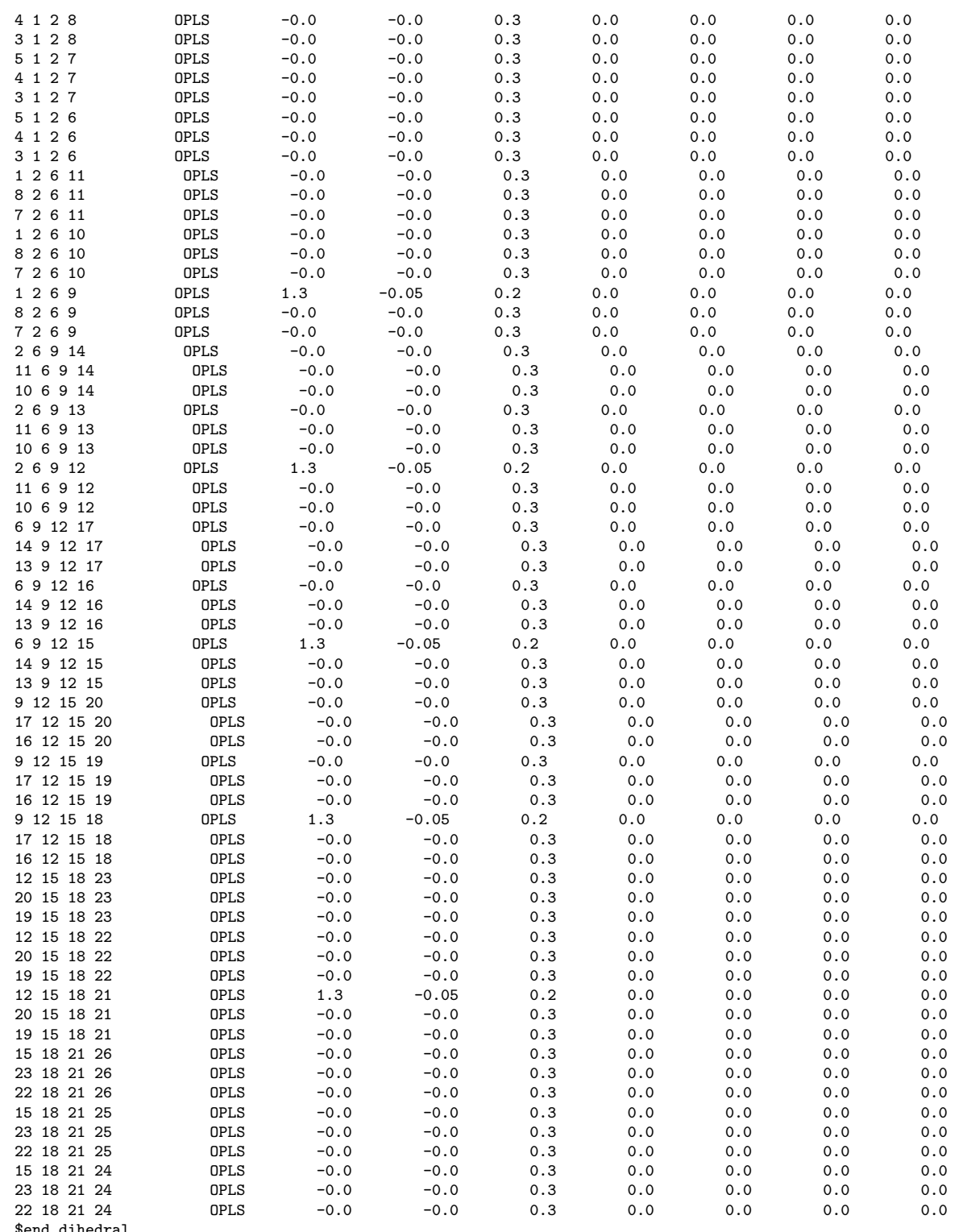

title $=$ octane_chloroform_therm

ljname $=$ octane.txt

outname $=$ outoctane

ncores $=6$

init $=$ yes

coolstep $=200$

nmol $=11000$

dens $=1.45$

temp $=298.15$

press $=1.0$

upbuf $=100$

accum $=$ no

vstep $=25000$

nstep $=4$

iprint $=1$
isave $=5000$

irdf $=0$

iratio $=10$ 
vratio $=10$

seed $=85513$

nsf $=10000$

sfint $=500$

DICE CBMC simulation input: octane.in

title $=$ octane - chloroform

ljname $=$ octane $\cdot$ txt

outname $=$ outoctane

ncores $=6$

init $=$ no

temp $=298.15$

press $=1.0$

accum $=$ no

vstep $=125000$

nstep $=4$

iprint $=1$

isave $=50$

irdf $=5$

iratio $=10$

vratio $=10$

seed $=208043$

flex $=$ octan

nsf $=10000$

sfint $=500$

equiphi $=$ yes

ntrialphi $=18$

pcbmc $=0.7$

dielectric $=4.81$

upbuf $=100$

sampling $=2$

\section{SI 5.2: neopentane}

Geometry and nonbonded parameters: neopentane.txt

\begin{tabular}{|c|c|c|c|c|c|c|}
\hline \\
\hline 17 & neopentane & generated with gr & & & & \\
\hline 1 & 1.0000 & 1.0000 & 0.0000 & -0.1800 & 0.0660 & 3.5000 \\
\hline 1 & -0.5400 & 1.0000 & 0.0000 & 0.0000 & 0.0660 & 3.5000 \\
\hline 1 & -1.0400 & 1.0000 & 1.4500 & -0.1800 & 0.0660 & 3.5000 \\
\hline 1 & -1.0400 & 2.2600 & -0.7200 & -0.1800 & 0.0660 & 3.5000 \\
\hline 1 & -1.0400 & -0.2600 & -0.7200 & -0.1800 & 0.0660 & 3.5000 \\
\hline 2 & 1.4000 & 1.8900 & 0.5100 & 0.0600 & 0.0300 & 2.5000 \\
\hline 2 & 1.4000 & 1.0000 & -1.0200 & 0.0600 & 0.0300 & 2.5000 \\
\hline 2 & 1.4000 & 0.1200 & 0.5100 & 0.0600 & 0.0300 & 2.5000 \\
\hline 2 & -0.6900 & 1.8900 & 1.9900 & 0.0600 & 0.0300 & 2.5000 \\
\hline 2 & -0.6900 & 0.1200 & 1.9900 & 0.0600 & 0.0300 & 2.5000 \\
\hline 2 & -2.1400 & 1.0000 & 1.4800 & 0.0600 & 0.0300 & 2.5000 \\
\hline 2 & -0.6900 & 3.1700 & -0.2300 & 0.0600 & 0.0300 & 2.5000 \\
\hline 2 & -2.1400 & 2.2800 & -0.7400 & 0.0600 & 0.0300 & 2.5000 \\
\hline 2 & -0.6900 & 2.2800 & -1.7600 & 0.0600 & 0.0300 & 2.5000 \\
\hline 2 & -0.6900 & -1.1700 & -0.2300 & 0.0600 & 0.0300 & 2.5000 \\
\hline 2 & -0.6900 & -0.2800 & -1.7600 & 0.0600 & 0.0300 & 2.5000 \\
\hline 2 & -2.1400 & -0.2800 & -0.7400 & 0.0600 & 0.0300 & 2.5000 \\
\hline
\end{tabular}

\begin{tabular}{|c|c|c|c|c|c|c|}
\hline & fragments & & & & & \\
\hline 1 & {$[1$} & 2 & 6 & 8 & 7 & ] $\mathrm{F}$ \\
\hline 2 & [ 2 & 3 & 5 & 4 & 1 & ] $\mathrm{F}$ \\
\hline 3 & {$[3$} & 2 & 9 & 10 & 11 & ] $\mathrm{F}$ \\
\hline 4 & [ 4 & 2 & 12 & 13 & 14 & ] $\mathrm{F}$ \\
\hline 5 & [ 5 & 2 & 15 & 17 & 16 & ] $\mathrm{F}$ \\
\hline
\end{tabular}

\$fragment connection

$\begin{array}{ll}1 & 2 \\ 2 & 4 \\ 2 & 5 \\ 2 & 3\end{array}$

end fragment connection

\$bond

$\begin{array}{lll}21 & 268.0 & 1.529\end{array}$ 


$\begin{array}{llll}3 & 2 & 268.0 & 1.529 \\ 4 & 2 & 268.0 & 1.529 \\ 5 & 2 & 268.0 & 1.529 \\ 6 & 1 & 340.0 & 1.09 \\ 7 & 1 & 340.0 & 1.09 \\ 8 & 1 & 340.0 & 1.09 \\ 93 & 340.0 & 1.09 \\ 103 & 340.0 & 1.09 \\ 113 & 340.0 & 1.09 \\ 12 & 4 & 340.0 & 1.09 \\ 13 & 4 & 340.0 & 1.09 \\ 14 & 4 & 340.0 & 1.09 \\ 15 & 5 & 340.0 & 1.09 \\ 16 & 5 & 340.0 & 1.09 \\ 17 & 5 & 340.0 & 1.09 \\ \text { \$end bond } & & \end{array}$

\$angle

$\begin{array}{lll}1 & 2 & 3 \\ 1 & 2 & 4\end{array}$

$\begin{array}{lll}1 & 2 & 4 \\ 1 & 2 & 5\end{array}$

216

217

218

$\begin{array}{lll}2 & 3 & 9 \\ 2 & 3 & 10\end{array}$

$\begin{array}{lll}2 & 3 & 10 \\ 2 & 3 & 11\end{array}$

$\begin{array}{lll}2 & 3 & 11 \\ 2 & 4 & 12\end{array}$

2413

2414

2515

$\begin{array}{lll}2 & 5 & 16\end{array}$

$\begin{array}{lll}2 & 5 & 17 \\ 15 & 5 & 16\end{array}$

13414

$\begin{array}{lll}15 & 5 & 17\end{array}$

$\begin{array}{lll}10 & 3 & 11 \\ 16 & 5 & 17\end{array}$

324

$\begin{array}{lll}3 & 2 & 4 \\ 9 & 3 & 10\end{array}$

325

$4 \quad 2 \quad 5$
$12 \quad 4 \quad 13$

$9 \begin{array}{lll}9 & 11\end{array}$

$\begin{array}{lll}7 & 1 & 8\end{array}$

617

12414

618

\$end angle

$\begin{array}{ccc}\text { harmonic } & 58.35 & 112.7 \\ \text { harmonic } & 58.35 & 112.7 \\ \text { harmonic } & 58.35 & 112.7 \\ \text { harmonic } & 37.5 & 110.7 \\ \text { harmonic } & 37.5 & 110.7 \\ \text { harmonic } & 37.5 & 110.7 \\ \text { harmonic } & 37.5 & 110.7 \\ \text { harmonic } & 37.5 & 110.7 \\ \text { harmonic } & 37.5 & 110.7 \\ \text { harmonic } & 37.5 & 110.7 \\ \text { harmonic } & 37.5 & 110.7 \\ \text { harmonic } & 37.5 & 110.7 \\ \text { harmonic } & 37.5 & 110.7 \\ \text { harmonic } & 37.5 & 110.7 \\ \text { harmonic } & 37.5 & 110.7 \\ \text { harmonic } & 33.0 & 107.8 \\ \text { harmonic } & 33.0 & 107.8 \\ \text { harmonic } & 33.0 & 107.8 \\ \text { harmonic } & 33.0 & 107.8 \\ \text { harmonic } & 33.0 & 107.8 \\ \text { harmonic } & 58.35 & 112.7 \\ \text { harmonic } & 33.0 & 107.8 \\ \text { harmonic } & 58.35 & 112.7 \\ \text { harmonic } & 58.35 & 112.7 \\ \text { harmonic } & 33.0 & 107.8 \\ \text { harmonic } & 33.0 & 107.8 \\ \text { harmonic } & 33.0 & 107.8 \\ \text { harmonic } & 33.0 & 107.8 \\ \text { harmonic } & 33.0 & 107.8 \\ \text { harmonic } & 33.0 & 107.8 \\ & & \end{array}$

\$dihedral

13423
9324

$\begin{array}{llll}9 & 324\end{array}$

$\begin{array}{llll}15 & 5 & 24 \\ 17 & 5 & 24\end{array}$

$\begin{array}{llll}16 & 5 & 2 & 3\end{array}$

$\begin{array}{llll}17 & 5 & 2 & 3\end{array}$

16524

9325

8123

$\begin{array}{llll}6 & 1 & 2 & 5 \\ 7 & 1 & 2 & 4\end{array}$

$\begin{array}{llll}7 & 1 & 2 & 4\end{array}$

12425

$\begin{array}{llll}10 & 3 & 2 & 1\end{array}$

$\begin{array}{llll}13 & 4 & 2 & 1\end{array}$

$\begin{array}{llll}11 & 3 & 2 & 4\end{array}$

$\begin{array}{llll}11 & 3 & 2 & 4 \\ 16 & 5 & 2 & 1\end{array}$

$\begin{array}{llll}16 & 5 & 2 & 1 \\ 12 & 4 & 2 & 3\end{array}$

10324

$\begin{array}{llll}17 & 5 & 2 & 1\end{array}$

14423

14425

11325

6123

932

12421

8125

$\begin{array}{llll}11 & 3 & 2 & 1\end{array}$

$\begin{array}{llll}81 & 124\end{array}$

$\begin{array}{llll}7 & 1 & 2 & 3\end{array}$

6124

13425

$\begin{array}{llll}15 & 5 & 2 & 3 \\ 14 & 4 & 2 & 1\end{array}$

\begin{tabular}{ccc} 
OPLS & -0.0 & -0.0 \\
OPLS & -0.0 & -0.0 \\
OPLS & -0.0 & -0.0 \\
OPLS & -0.0 & -0.0 \\
OPLS & -0.0 & -0.0 \\
OPLS & -0.0 & -0.0 \\
OPLS & -0.0 & -0.0 \\
OPLS & -0.0 & -0.0 \\
OPLS & -0.0 & -0.0 \\
OPLS & -0.0 & -0.0 \\
OPLS & -0.0 & -0.0 \\
OPLS & -0.0 & -0.0 \\
OPLS & -0.0 & -0.0 \\
OPLS & -0.0 & -0.0 \\
OPLS & -0.0 & -0.0 \\
OPLS & -0.0 & -0.0 \\
OPLS & -0.0 & -0.0 \\
OPLS & -0.0 & -0.0 \\
OPLS & -0.0 & -0.0 \\
OPLS & -0.0 & -0.0 \\
OPLS & -0.0 & -0.0 \\
OPLS & -0.0 & -0.0 \\
OPLS & -0.0 & -0.0 \\
OPLS & -0.0 & -0.0 \\
OPLS & -0.0 & -0.0 \\
OPLS & -0.0 & -0.0 \\
OPLS & -0.0 & -0.0 \\
OPLS & -0.0 & -0.0 \\
OPLS & -0.0 & -0.0 \\
OPLS & -0.0 & -0.0 \\
OPLS & -0.0 & -0.0 \\
OPLS & -0.0 & -0.0 \\
OPLS & -0.0 & -0.0 \\
OPLS & -0.0 & -0.0 \\
OPLS & -0.0 & -0.0 \\
OPLS & -0.0 & -0.0 \\
\hline & &
\end{tabular}

0.3
0.3
0.3
0.3
0.3
0.3
0.3
0.3
0.3
0.3
0.3
0.3
0.3
0.3
0.3
0.3
0.3
0.3
0.3
0.3
0.3
0.3
0.3
0.3
0.3
0.3
0.3
0.3
0.3
0.3
0.3
0.3
0.3
0.3
0.3
0.3

$\begin{array}{ccc}0.0 & 0.0 & 0.0 \\ 0.0 & 0.0 & 0.0 \\ 0.0 & 0.0 & 0.0 \\ 0.0 & 0.0 & 0.0 \\ 0.0 & 0.0 & 0.0 \\ 0.0 & 0.0 & 0.0 \\ 0.0 & 0.0 & 0.0 \\ 0.0 & 0.0 & 0.0 \\ 0.0 & 0.0 & 0.0 \\ 0.0 & 0.0 & 0.0 \\ 0.0 & 0.0 & 0.0 \\ 0.0 & 0.0 & 0.0 \\ 0.0 & 0.0 & 0.0 \\ 0.0 & 0.0 & 0.0 \\ 0.0 & 0.0 & 0.0 \\ 0.0 & 0.0 & 0.0 \\ 0.0 & 0.0 & 0.0 \\ 0.0 & 0.0 & 0.0 \\ 0.0 & 0.0 & 0.0 \\ 0.0 & 0.0 & 0.0 \\ 0.0 & 0.0 & 0.0 \\ 0.0 & 0.0 & 0.0 \\ 0.0 & 0.0 & 0.0 \\ 0.0 & 0.0 & 0.0 \\ 0.0 & 0.0 & 0.0 \\ 0.0 & 0.0 & 0.0 \\ 0.0 & 0.0 & 0.0 \\ 0.0 & 0.0 & 0.0 \\ 0.0 & 0.0 & 0.0 \\ 0.0 & 0.0 & 0.0 \\ 0.0 & 0.0 & 0.0 \\ 0.0 & 0.0 & 0.0 \\ 0.0 & 0.0 & 0.0 \\ 0.0 & 0.0 & 0.0 \\ 0.0 & 0.0 & 0.0 \\ 0.0 & 0.0 & 0.0 \\ 0 & & \end{array}$


DICE CBMC simulation input: neopentane.in

title $=$ neopentane $e_{-g a s}$

ljname $=$ neopentane.txt

outname $=$ outneopentane

ncores $=4$

init $=$ yes

coolstep $=0$

nmol $=1$

dens $=0.001$

temp $=298.15$

press $=1.0$

upbuf $=99999999$

accum $=$ no

vstep $=0$

nstep $=10000000$

iprint $=1$

isave $=100$

irdf $=0$

iratio $=10$

vratio $=10$

seed $=70016$

flex $=$ neopentan

pcbmc $=1.0$

equiphi $=$ yes

ntrialphi $=12$

nsf $=10000$

sfint $=500$

\section{SI 5.3: 4-ethylheptane}

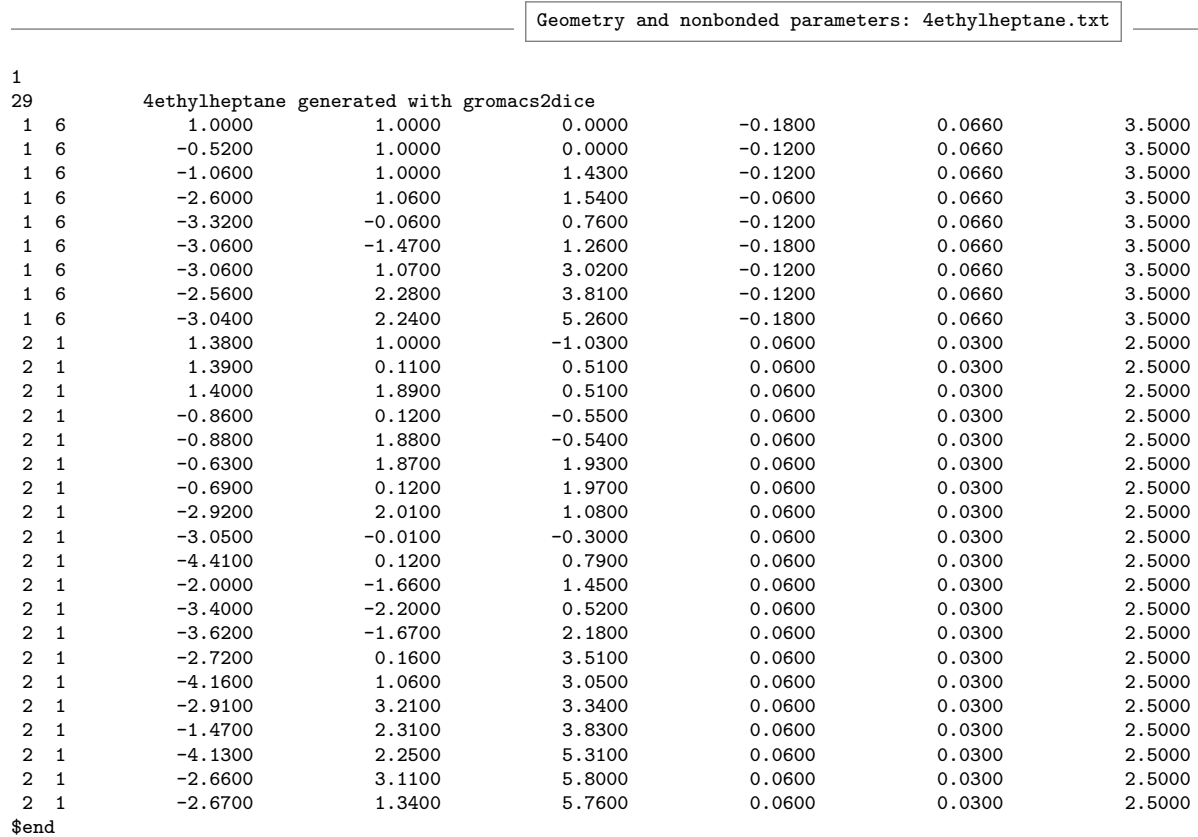

\begin{tabular}{|c|c|c|c|c|c|c|}
\hline \multicolumn{7}{|c|}{ \$atoms fragments } \\
\hline 1 & {$[1$} & 2 & 11 & 12 & 10 & ] $M$ \\
\hline 2 & {$[2$} & 3 & 1 & 14 & 13 & ] $M$ \\
\hline 3 & {$[3$} & 4 & 2 & 16 & 15 & ] $M$ \\
\hline 4 & {$[4$} & 7 & 5 & 3 & 17 & ] $M$ \\
\hline 5 & {$[5$} & 6 & 4 & 19 & 18 & ] $\mathrm{M}$ \\
\hline 6 & {$[6$} & 5 & 22 & 20 & 21 & ] $M$ \\
\hline
\end{tabular}




\begin{tabular}{|c|c|c|c|c|c|c|}
\hline 7 & [ 7 & 8 & 4 & 23 & 24 & ] $M$ \\
\hline 8 & [ 8 & 9 & 7 & 26 & 25 & ] $\mathrm{M}$ \\
\hline 9 & [ 9 & 8 & 28 & 29 & 27 & ] $M$ \\
\hline
\end{tabular}

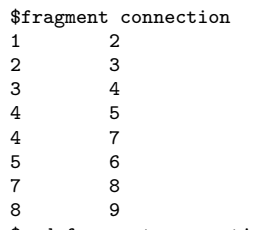

\$end fragment connection

\begin{tabular}{|c|c|c|}
\hline \multicolumn{3}{|c|}{ \$bond } \\
\hline 21 & 268.0 & 1.529 \\
\hline 32 & 268.0 & 1.529 \\
\hline 43 & 268.0 & 1.529 \\
\hline 54 & 268.0 & 1.529 \\
\hline 65 & 268.0 & 1.529 \\
\hline 74 & 268.0 & 1.529 \\
\hline 87 & 268.0 & 1.529 \\
\hline 98 & 268.0 & 1.529 \\
\hline 101 & 340.0 & 1.09 \\
\hline 111 & 340.0 & 1.09 \\
\hline 121 & 340.0 & 1.09 \\
\hline 132 & 340.0 & 1.09 \\
\hline 142 & 340.0 & 1.09 \\
\hline 153 & 340.0 & 1.09 \\
\hline 163 & 340.0 & 1.09 \\
\hline 174 & 340.0 & 1.09 \\
\hline 185 & 340.0 & 1.09 \\
\hline 195 & 340.0 & 1.09 \\
\hline 206 & 340.0 & 1.09 \\
\hline 216 & 340.0 & 1.09 \\
\hline 226 & 340.0 & 1.09 \\
\hline 237 & 340.0 & 1.09 \\
\hline 247 & 340.0 & 1.09 \\
\hline 258 & 340.0 & 1.09 \\
\hline 268 & 340.0 & 1.09 \\
\hline 279 & 340.0 & 1.09 \\
\hline 289 & 340.0 & 1.09 \\
\hline 299 & 340.0 & 1.09 \\
\hline
\end{tabular}

\begin{tabular}{|c|c|c|c|}
\hline \multicolumn{4}{|l|}{ \$angle } \\
\hline 123 & harmonic & 58.35 & 112.7 \\
\hline 234 & harmonic & 58.35 & 112.7 \\
\hline 345 & harmonic & 58.35 & 112.7 \\
\hline 456 & harmonic & 58.35 & 112.7 \\
\hline 347 & harmonic & 58.35 & 112.7 \\
\hline 478 & harmonic & 58.35 & 112.7 \\
\hline 789 & harmonic & 58.35 & 112.7 \\
\hline $\begin{array}{lll}2 & 1 & 10\end{array}$ & harmonic & 37.5 & 110.7 \\
\hline $\begin{array}{lll}2 & 1 & 11\end{array}$ & harmonic & 37.5 & 110.7 \\
\hline $\begin{array}{lll}2 & 1 & 12\end{array}$ & harmonic & 37.5 & 110.7 \\
\hline 1213 & harmonic & 37.5 & 110.7 \\
\hline $\begin{array}{lll}12 & 14\end{array}$ & harmonic & 37.5 & 110.7 \\
\hline $23 \quad 15$ & harmonic & 37.5 & 110.7 \\
\hline 23316 & harmonic & 37.5 & 110.7 \\
\hline $\begin{array}{lll}3 & 4 & 17\end{array}$ & harmonic & 37.5 & 110.7 \\
\hline $\begin{array}{lll}4 & 5 & 18\end{array}$ & harmonic & 37.5 & 110.7 \\
\hline $\begin{array}{lll}4 & 5 & 19\end{array}$ & harmonic & 37.5 & 110.7 \\
\hline 5620 & harmonic & 37.5 & 110.7 \\
\hline 5621 & harmonic & 37.5 & 110.7 \\
\hline $\begin{array}{lll}5 & 6 & 22\end{array}$ & harmonic & 37.5 & 110.7 \\
\hline 4723 & harmonic & 37.5 & 110.7 \\
\hline $4 \quad 7 \quad 24$ & harmonic & 37.5 & 110.7 \\
\hline 7825 & harmonic & 37.5 & 110.7 \\
\hline $\begin{array}{lll}78 & 26\end{array}$ & harmonic & 37.5 & 110.7 \\
\hline $\begin{array}{lll}8 & 9 & 27\end{array}$ & harmonic & 37.5 & 110.7 \\
\hline 8928 & harmonic & 37.5 & 110.7 \\
\hline 8929 & harmonic & 37.5 & 110.7 \\
\hline $23 \quad 7 \quad 24$ & harmonic & 33.0 & 107.8 \\
\hline 9825 & harmonic & 37.5 & 110.7 \\
\hline $\begin{array}{lll}3 & 2 & 14\end{array}$ & harmonic & 37.5 & 110.7 \\
\hline $13 \quad 2 \quad 14$ & harmonic & 33.0 & 107.8 \\
\hline 20621 & harmonic & 33.0 & 107.8 \\
\hline 547 & harmonic & 58.35 & 112.7 \\
\hline 21622 & harmonic & 33.0 & 107.8 \\
\hline 20622 & harmonic & 33.0 & 107.8 \\
\hline $\begin{array}{lll}6 & 5 & 19\end{array}$ & harmonic & 37.5 & 110.7 \\
\hline $\begin{array}{lll}28 & 9 & 29\end{array}$ & harmonic & 33.0 & 107.8 \\
\hline 15316 & harmonic & 33.0 & 107.8 \\
\hline 8723 & harmonic & 37.5 & 110.7 \\
\hline
\end{tabular}




\begin{tabular}{|c|c|c|c|}
\hline $\begin{array}{lll}27 & 9 & 29\end{array}$ & harmonic & 33.0 & 107.8 \\
\hline $\begin{array}{lll}11 & 1 & 12\end{array}$ & harmonic & 33.0 & 107.8 \\
\hline $\begin{array}{llll}7 & 4 & 17\end{array}$ & harmonic & 37.5 & 110.7 \\
\hline 25826 & harmonic & 33.0 & 107.8 \\
\hline 27928 & harmonic & 33.0 & 107.8 \\
\hline $\begin{array}{lll}10 & 1 & 12\end{array}$ & harmonic & 33.0 & 107.8 \\
\hline 10111 & harmonic & 33.0 & 107.8 \\
\hline $\begin{array}{lll}32 & 13\end{array}$ & harmonic & 37.5 & 110.7 \\
\hline $\begin{array}{lll}18 & 5 & 19\end{array}$ & harmonic & 33.0 & 107.8 \\
\hline 9826 & harmonic & 37.5 & 110.7 \\
\hline 4316 & harmonic & 37.5 & 110.7 \\
\hline 8724 & harmonic & 37.5 & 110.7 \\
\hline 4315 & harmonic & 37.5 & 110.7 \\
\hline $\begin{array}{lll}6 & 5 & 18\end{array}$ & harmonic & 37.5 & 110.7 \\
\hline $\begin{array}{lll}54 & 17\end{array}$ & harmonic & 37.5 & 110.7 \\
\hline
\end{tabular}

\$dihedral

$\begin{array}{llll}4 & 3 & 2 & 1\end{array}$

$\begin{array}{llll}7 & 4 & 5 & 6\end{array}$

6543

5432

8743

$\begin{array}{llll}11 & 1 & 2 & 3\end{array}$

$\begin{array}{llll}24 & 7 & 8 & 9 \\ 23 & 7 & 8 & 9\end{array}$

23743

17456

$\begin{array}{llll}29 & 9 & 8 & 7\end{array}$

19547

$\begin{array}{llll}26 & 8 & 7 & 4\end{array}$

17478

$\begin{array}{llll}25 & 8 & 7 & 4\end{array}$

$\begin{array}{llll}21 & 6 & 5 & 4 \\ 24 & 7 & 4 & 5\end{array}$

18547

12123

23745

22654

16347

$\begin{array}{llll}19 & 5 & 4 & 3 \\ 28 & 9 & 8 & 7\end{array}$

14234

$\begin{array}{llll}24 & 7 & 4 & 3 \\ 13 & 2 & 3 & 4\end{array}$

$\begin{array}{llll}15 & 3 & 2 & 1\end{array}$

20654

$\begin{array}{llll}10 & 1 & 2 & 3 \\ 18 & 5 & 4 & 3\end{array}$

$\begin{array}{llll}18 & 5 & 4 & 3\end{array}$

$\begin{array}{llll}16 & 3 & 4 & 5\end{array}$

16321

$\begin{array}{llll}27 & 9 & 8 & 7\end{array}$

$\begin{array}{llll}15 & 3 & 4 & 5\end{array}$

$\begin{array}{llll}15 & 5 & 4 & 17\end{array}$

$\begin{array}{llll}18 & 5 & 4 & 17 \\ 14 & 2 & 1 & 12\end{array}$

$\begin{array}{llll}15 & 3 & 2 & 13\end{array}$

$\begin{array}{llll}29 & 9 & 8 & 25\end{array}$

$\begin{array}{llll}16 & 3 & 2 & 14\end{array}$

$\begin{array}{llll}22 & 6 & 5 & 18\end{array}$

$\begin{array}{llll}13 & 2 & 1 & 10\end{array}$

$\begin{array}{llll}7 & 9 & 8 & 26\end{array}$

$\begin{array}{llll}3 & 7 & 4 & 17\end{array}$

$\begin{array}{llll}17 & 4 & 3 & 16\end{array}$

$\begin{array}{llll}20 & 6 & 5 & 18\end{array}$

$\begin{array}{llll}27 & 9 & 8 & 25 \\ 14 & 2 & 1 & 10\end{array}$

$\begin{array}{llll}29 & 9 & 8 & 26\end{array}$

247417

$1743 \quad 15$

$\begin{array}{llll}16 & 3 & 2 & 13\end{array}$

$\begin{array}{llll}25 & 8 & 7 & 23\end{array}$

$\begin{array}{llll}15 & 3 & 2 & 14\end{array}$

$\begin{array}{llll}22 & 6 & 5 & 19\end{array}$

$\begin{array}{llll}25 & 8 & 7 & 24\end{array}$

$\begin{array}{llll}28 & 9 & 8 & 25\end{array}$

$20 \quad 6519$

$\begin{array}{llll}21 & 6 & 5 & 18\end{array}$

$\begin{array}{llll}3 & 2 & 1 & 12\end{array}$

$\begin{array}{llll}14 & 2 & 1 & 11\end{array}$

$\begin{array}{llll}21 & 6 & 5 & 19\end{array}$

$\begin{array}{llll}19 & 5 & 4 & 17 \\ 26 & 8 & 7 & 24\end{array}$

\begin{tabular}{|c|c|c|}
\hline OPLS & 1.3 & -0.05 \\
\hline OPLS & 1.3 & -0.05 \\
\hline OPLS & 1.3 & -0.05 \\
\hline OPLS & 1.3 & -0.05 \\
\hline OPLS & 1.3 & -0.05 \\
\hline OPLS & 1.3 & -0.05 \\
\hline OPLS & 1.3 & -0.05 \\
\hline OPLS & 1.3 & -0.05 \\
\hline OPLS & -0.0 & -0.0 \\
\hline OPLS & -0.0 & -0.0 \\
\hline OPLS & -0.0 & -0.0 \\
\hline OPLS & -0.0 & -0.0 \\
\hline OPLS & -0.0 & -0.0 \\
\hline OPLS & -0.0 & -0.0 \\
\hline OPLS & -0.0 & -0.0 \\
\hline OPLS & -0.0 & -0.0 \\
\hline OPLS & -0.0 & -0.0 \\
\hline OPLS & -0.0 & -0.0 \\
\hline OPLS & -0.0 & -0.0 \\
\hline OPLS & -0.0 & -0.0 \\
\hline OPLS & -0.0 & -0.0 \\
\hline OPLS & -0.0 & -0.0 \\
\hline OPLS & -0.0 & -0.0 \\
\hline OPLS & -0.0 & -0.0 \\
\hline OPLS & -0.0 & -0.0 \\
\hline OPLS & -0.0 & -0.0 \\
\hline OPLS & -0.0 & -0.0 \\
\hline OPLS & -0.0 & -0.0 \\
\hline OPLS & -0.0 & -0.0 \\
\hline OPLS & -0.0 & -0.0 \\
\hline OPLS & -0.0 & -0.0 \\
\hline OPLS & -0.0 & -0.0 \\
\hline OPLS & -0.0 & -0.0 \\
\hline OPLS & -0.0 & -0.0 \\
\hline OPLS & -0.0 & -0.0 \\
\hline OPLS & -0.0 & -0.0 \\
\hline OPLS & -0.0 & -0.0 \\
\hline OPLS & -0.0 & -0.0 \\
\hline OPLS & -0.0 & -0.0 \\
\hline OPLS & -0.0 & -0.0 \\
\hline OPLS & -0.0 & -0.0 \\
\hline OPLS & -0.0 & -0.0 \\
\hline OPLS & -0.0 & -0.0 \\
\hline OPLS & -0.0 & -0.0 \\
\hline OPLS & -0.0 & -0.0 \\
\hline OPLS & -0.0 & -0.0 \\
\hline OPLS & -0.0 & -0.0 \\
\hline OPLS & -0.0 & -0.0 \\
\hline OPLS & -0.0 & -0.0 \\
\hline OPLS & -0.0 & -0.0 \\
\hline OPLS & -0.0 & -0.0 \\
\hline OPLS & -0.0 & -0.0 \\
\hline OPLS & -0.0 & -0.0 \\
\hline OPLS & -0.0 & -0.0 \\
\hline OPLS & -0.0 & -0.0 \\
\hline OPLS & -0.0 & -0.0 \\
\hline OPLS & -0.0 & -0.0 \\
\hline OPLS & -0.0 & -0.0 \\
\hline OPLS & -0.0 & -0.0 \\
\hline OPLS & -0.0 & -0.0 \\
\hline OPLS & -0.0 & -0.0 \\
\hline OPLS & -0.0 & -0.0 \\
\hline OPLS & -0.0 & -0.0 \\
\hline OPLS & -0.0 & -0.0 \\
\hline OPLS & -0.0 & -0.0 \\
\hline OPLS & -0.0 & -0.0 \\
\hline OPLS & -0.0 & -0.0 \\
\hline OPLS & -0.0 & -0.0 \\
\hline OPLS & -0.0 & -0.0 \\
\hline
\end{tabular}

\begin{tabular}{|c|c|c|c|}
\hline 0.2 & 0.0 & 0.0 & 0.0 \\
\hline 0.2 & 0.0 & 0.0 & 0.0 \\
\hline 0.2 & 0.0 & 0.0 & 0.0 \\
\hline 0.2 & 0.0 & 0.0 & 0.0 \\
\hline 0.2 & 0.0 & 0.0 & 0.0 \\
\hline 0.2 & 0.0 & 0.0 & 0.0 \\
\hline 0.2 & 0.0 & 0.0 & 0.0 \\
\hline 0.2 & 0.0 & 0.0 & 0.0 \\
\hline 0.3 & 0.0 & 0.0 & 0.0 \\
\hline 0.3 & 0.0 & 0.0 & 0.0 \\
\hline 0.3 & 0.0 & 0.0 & 0.0 \\
\hline 0.3 & 0.0 & 0.0 & 0.0 \\
\hline 0.3 & 0.0 & 0.0 & 0.0 \\
\hline 0.3 & 0.0 & 0.0 & 0.0 \\
\hline 0.3 & 0.0 & 0.0 & 0.0 \\
\hline 0.3 & 0.0 & 0.0 & 0.0 \\
\hline 0.3 & 0.0 & 0.0 & 0.0 \\
\hline 0.3 & 0.0 & 0.0 & 0.0 \\
\hline 0.3 & 0.0 & 0.0 & 0.0 \\
\hline 0.3 & 0.0 & 0.0 & 0.0 \\
\hline 0.3 & 0.0 & 0.0 & 0.0 \\
\hline 0.3 & 0.0 & 0.0 & 0.0 \\
\hline 0.3 & 0.0 & 0.0 & 0.0 \\
\hline 0.3 & 0.0 & 0.0 & 0.0 \\
\hline 0.3 & 0.0 & 0.0 & 0.0 \\
\hline 0.3 & 0.0 & 0.0 & 0.0 \\
\hline 0.3 & 0.0 & 0.0 & 0.0 \\
\hline 0.3 & 0.0 & 0.0 & 0.0 \\
\hline 0.3 & 0.0 & 0.0 & 0.0 \\
\hline 0.3 & 0.0 & 0.0 & 0.0 \\
\hline 0.3 & 0.0 & 0.0 & 0.0 \\
\hline 0.3 & 0.0 & 0.0 & 0.0 \\
\hline 0.3 & 0.0 & 0.0 & 0.0 \\
\hline 0.3 & 0.0 & 0.0 & 0.0 \\
\hline 0.3 & 0.0 & 0.0 & 0.0 \\
\hline 0.3 & 0.0 & 0.0 & 0.0 \\
\hline 0.3 & 0.0 & 0.0 & 0.0 \\
\hline 0.3 & 0.0 & 0.0 & 0.0 \\
\hline 0.3 & 0.0 & 0.0 & 0.0 \\
\hline 0.3 & 0.0 & 0.0 & 0.0 \\
\hline 0.3 & 0.0 & 0.0 & 0.0 \\
\hline 0.3 & 0.0 & 0.0 & 0.0 \\
\hline 0.3 & 0.0 & 0.0 & 0.0 \\
\hline 0.3 & 0.0 & 0.0 & 0.0 \\
\hline 0.3 & 0.0 & 0.0 & 0.0 \\
\hline 0.3 & 0.0 & 0.0 & 0.0 \\
\hline 0.3 & 0.0 & 0.0 & 0.0 \\
\hline 0.3 & 0.0 & 0.0 & 0.0 \\
\hline 0.3 & 0.0 & 0.0 & 0.0 \\
\hline 0.3 & 0.0 & 0.0 & 0.0 \\
\hline 0.3 & 0.0 & 0.0 & 0.0 \\
\hline 0.3 & 0.0 & 0.0 & 0.0 \\
\hline 0.3 & 0.0 & 0.0 & 0.0 \\
\hline 0.3 & 0.0 & 0.0 & 0.0 \\
\hline 0.3 & 0.0 & 0.0 & 0.0 \\
\hline 0.3 & 0.0 & 0.0 & 0.0 \\
\hline 0.3 & 0.0 & 0.0 & 0.0 \\
\hline 0.3 & 0.0 & 0.0 & 0.0 \\
\hline 0.3 & 0.0 & 0.0 & 0.0 \\
\hline 0.3 & 0.0 & 0.0 & 0.0 \\
\hline 0.3 & 0.0 & 0.0 & 0.0 \\
\hline 0.3 & 0.0 & 0.0 & 0.0 \\
\hline 0.3 & 0.0 & 0.0 & 0.0 \\
\hline 0.3 & 0.0 & 0.0 & 0.0 \\
\hline 0.3 & 0.0 & 0.0 & 0.0 \\
\hline 0.3 & 0.0 & 0.0 & 0.0 \\
\hline 0.3 & 0.0 & 0.0 & 0.0 \\
\hline 0.3 & 0.0 & 0.0 & 0.0 \\
\hline 0.3 & 0.0 & 0.0 & 0.0 \\
\hline
\end{tabular}




\begin{tabular}{|c|c|c|c|c|c|c|c|}
\hline $\begin{array}{llll}13 & 2 & 1 & 11\end{array}$ & OPLS & -0.0 & -0.0 & 0.3 & 0.0 & 0.0 & 0.0 \\
\hline $\begin{array}{llll}28 & 9 & 8 & 26\end{array}$ & OPLS & -0.0 & -0.0 & 0.3 & 0.0 & 0.0 & 0.0 \\
\hline $\begin{array}{llll}26 & 8 & 7 & 23\end{array}$ & OPLS & -0.0 & -0.0 & 0.3 & 0.0 & 0.0 & 0.0 \\
\hline
\end{tabular}

DICE CBMC simulation input: 4ethylheptane.in

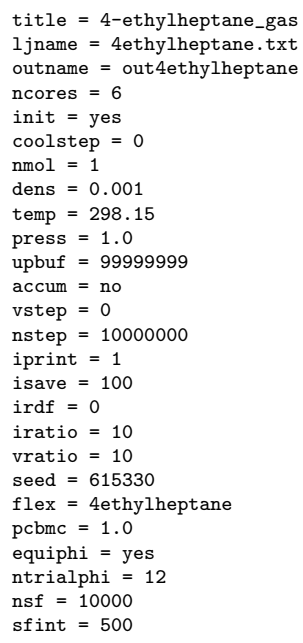

\section{SI 5.4: DCE}

Geometry and nonbonded parameters: dce.txt

\begin{tabular}{|c|c|c|c|c|c|c|}
\hline 8 & DCE gener & with $\varepsilon$ & acs2dice & & & \\
\hline 117 & 0.055 & 0.0 & -2.18 & -0.200 & 0.3 & 3.4 \\
\hline 26 & -0.584 & 0.0 & -0.494 & -0.006 & 0.066 & 3.5 \\
\hline 31 & -1.193 & 0.89 & -0.336 & 0.103 & 0.03 & 2.5 \\
\hline 31 & -1.193 & -0.89 & -0.336 & 0.103 & 0.03 & 2.5 \\
\hline 26 & 0.584 & 0.0 & 0.494 & -0.006 & 0.066 & 3.5 \\
\hline 31 & 1.193 & -0.89 & 0.336 & 0.103 & 0.03 & 2.5 \\
\hline 31 & 1.193 & 0.89 & 0.336 & 0.103 & 0.03 & 2.5 \\
\hline 117 & -0.055 & 0.0 & 2.18 & -0.200 & 0.3 & 3.4 \\
\hline \$end & & & & & & \\
\hline
\end{tabular}

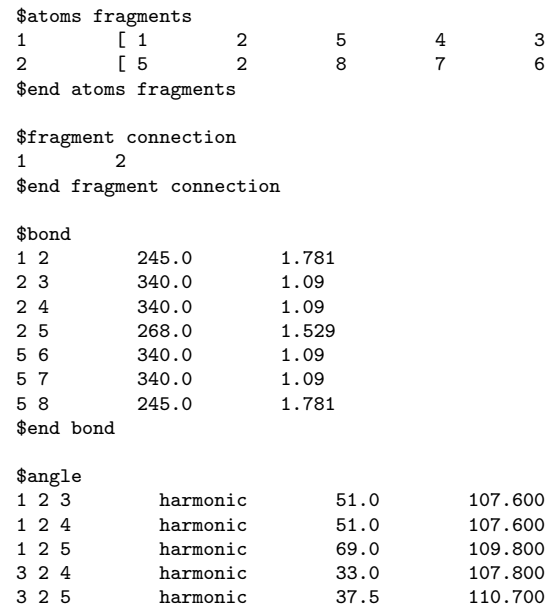




\begin{tabular}{|c|c|c|c|c|c|c|c|c|}
\hline 425 & harmonic & 37.5 & \multicolumn{2}{|c|}{110.700} & & & & \\
\hline 256 & harmonic & 37.5 & \multicolumn{2}{|c|}{110.700} & & & & \\
\hline 257 & harmonic & 37.5 & \multicolumn{2}{|c|}{110.700} & & & & \\
\hline 258 & harmonic & 69.0 & \multicolumn{2}{|c|}{109.800} & & & & \\
\hline 657 & harmonic & 33.0 & \multirow{2}{*}{\multicolumn{2}{|c|}{$\begin{array}{l}107.800 \\
107.600\end{array}$}} & & & & \\
\hline 658 & harmonic & 51.0 & & & & & & \\
\hline 758 & harmonic & 51.0 & \multicolumn{2}{|c|}{107.600} & & & & \\
\hline \multicolumn{9}{|c|}{ \$end angle } \\
\hline \multicolumn{9}{|l|}{ \$dihedral } \\
\hline 1256 & OPLS & 0.0 & 0.0 & 0.4 & 0.0 & 0.0 & 0.0 & 0.0 \\
\hline 1257 & OPLS & 0.0 & 0.0 & 0.4 & 0.0 & 0.0 & 0.0 & 0.0 \\
\hline 1258 & OPLS & -0.25 & 0.0 & 0.0 & 0.0 & 0.0 & 0.0 & 0.0 \\
\hline 3256 & OPLS & 0.0 & 0.0 & 0.318 & 0.0 & 0.0 & 0.0 & 0.0 \\
\hline 3257 & OPLS & 0.0 & 0.0 & 0.318 & 0.0 & 0.0 & 0.0 & 0.0 \\
\hline 3258 & OPLS & 0.0 & 0.0 & 0.4 & 0.0 & 0.0 & 0.0 & 0.0 \\
\hline 4256 & OPLS & 0.0 & 0.0 & 0.318 & 0.0 & 0.0 & 0.0 & 0.0 \\
\hline 4257 & OPLS & 0.0 & 0.0 & 0.318 & 0.0 & 0.0 & 0.0 & 0.0 \\
\hline 4258 & OPLS & 0.0 & 0.0 & 0.4 & 0.0 & 0.0 & 0.0 & 0.0 \\
\hline \$end dih & & & & & & & & \\
\hline
\end{tabular}

DICE thermalization input: dce.ter

title $=12$-dichloroethane _therm

ljname $=$ DCE. txt

outname $=$ outdce

ncores $=8$

init $=$ yes

coolstep $=250$

dens $=1.246$

temp $=298.15$

press $=1.0$

accum $=$ no

vstep $=50000$

nstep $=4$

iprint $=1$

isave $=100$

irdf $=0$

iratio $=10$

vratio $=10$

seed $=73095$

DICE CBMC simulation input: dce.in

title $=12$-dichloroethane

ljname $=$ DCE. txt

outname $=$ outdce

ncores $=8$

init $=$ no

$\mathrm{nmol}=300$

dens $=1.246$

temp $=298.15$

press $=1.0$

ntrialphi $=32$

pcbmc $=0.7$

accum $=$ yes

vstep $=500000$

nstep $=4$

iprint $=1$

isave $=100$

irdf $=5$

iratio $=10$

iratio $=10$
vratio $=10$

seed $=62930$

nsf $=10000$

sfint $=500$

\section{SI 5.5: $\quad$ SubPC}

Geometry and nonbonded parameters: subpc.txt

2

94 subpc

$\begin{array}{lllllll}16 & 12.873058 & -1.455333 & -0.10028 & -0.107163 & 0.070 & 3.55 \\ 16 & 12.450469 & -0.40574 & -0.918682 & -0.013610 & 0.070 & 3.55\end{array}$ 


\begin{tabular}{|c|c|c|c|c|c|c|}
\hline 16 & 11.111274 & -0.040717 & -0.95111 & -0.244780 & 0.070 & 3.55 \\
\hline 16 & 10.164149 & -0.72226 & -0.173535 & 0.247184 & 0.070 & 3.55 \\
\hline 16 & 10.602464 & -1.771343 & 0.648846 & -0.205367 & 0.070 & 3.55 \\
\hline 16 & 11.940369 & -2.134176 & 0.685318 & -0.039676 & 0.070 & 3.55 \\
\hline 16 & 8.724595 & -0.376086 & -0.226869 & -0.159197 & 0.070 & 3.55 \\
\hline 16 & 7.749642 & -1.342247 & -0.168346 & -0.100388 & 0.070 & 3.55 \\
\hline 16 & 6.355625 & -1.055152 & -0.210916 & -0.130470 & 0.070 & 3.55 \\
\hline 16 & 5.871729 & 0.227112 & -0.315144 & 0.001834 & 0.070 & 3.55 \\
\hline 16 & 6.848141 & 1.28219 & -0.375392 & 0.261900 & 0.070 & 3.55 \\
\hline 16 & 8.260771 & 0.983688 & -0.334927 & 0.314848 & 0.070 & 3.55 \\
\hline 27 & 9.023488 & 2.073995 & -0.388912 & -0.417626 & 0.170 & 3.25 \\
\hline & 8.015312 & 3.359862 & -0.488793 & 0.363370 & 0.250 & 3.70 \\
\hline 27 & 6.575639 & 2.579246 & -0.46261 & -0.404432 & 0.170 & 3.25 \\
\hline 16 & 4.479463 & 0.523145 & -0.365382 & -0.027096 & 0.066 & 3.50 \\
\hline 16 & 3.293876 & 0.778781 & -0.410667 & -0.207739 & 0.066 & 3.50 \\
\hline 16 & 1.901464 & 1.099201 & -0.469095 & 0.264960 & 0.070 & 3.55 \\
\hline 16 & 0.940639 & 0.095441 & -0.322352 & -0.162689 & 0.070 & 3.55 \\
\hline 16 & -0.403209 & 0.446969 & -0.40799 & -0.062332 & 0.070 & 3.55 \\
\hline 16 & -0.793382 & 1.800838 & -0.613447 & -0.095612 & 0.070 & 3.55 \\
\hline 16 & 0.171776 & 2.804514 & -0.730271 & -0.053265 & 0.070 & 3.55 \\
\hline 16 & 1.50567 & 2.446456 & -0.670607 & -0.172941 & 0.070 & 3.55 \\
\hline 16 & -2.244765 & 1.840651 & -0.557493 & 0.511584 & 0.070 & 3.55 \\
\hline 27 & -2.641177 & 0.540115 & -0.474638 & -0.558906 & 0.170 & 3.25 \\
\hline 16 & -1.623275 & -0.327065 & -0.225768 & 0.496432 & 0.070 & 3.55 \\
\hline 45 & -4.075551 & 0.140371 & -0.389085 & 1.130301 & 0.095 & 3.54 \\
\hline 58 & -4.584662 & -0.207829 & -1.688693 & -0.708028 & 0.170 & 3.07 \\
\hline 16 & -5.884035 & -0.612796 & -1.846975 & 0.474011 & 0.070 & 3.55 \\
\hline 16 & -6.21876 & -1.965521 & -1.760628 & -0.228852 & 0.070 & 3.55 \\
\hline 16 & -7.531986 & -2.372462 & -1.961545 & -0.125772 & 0.070 & 3.55 \\
\hline 16 & -8.555114 & -1.459352 & -2.254385 & -0.109690 & 0.070 & 3.55 \\
\hline 16 & -8.195923 & -0.111805 & -2.34186 & -0.114218 & 0.070 & 3.55 \\
\hline 16 & -6.882152 & 0.311246 & -2.142166 & -0.243745 & 0.070 & 3.55 \\
\hline 27 & -1.814002 & -1.515645 & 0.361202 & -0.581988 & 0.170 & 3.25 \\
\hline 16 & -3.02656 & -1.767506 & 0.859067 & 0.478463 & 0.070 & 3.55 \\
\hline 16 & -3.43566 & -2.667622 & 1.931297 & -0.094464 & 0.070 & 3.55 \\
\hline 16 & -4.693238 & -2.205008 & 2.407274 & -0.001013 & 0.070 & 3.55 \\
\hline 16 & -5.041822 & -1.027278 & 1.621189 & 0.363530 & 0.070 & 3.55 \\
\hline 27 & -4.092548 & -0.941366 & 0.649862 & -0.434141 & 0.170 & 3.25 \\
\hline 16 & -5.307755 & -2.813214 & 3.501783 & -0.091993 & 0.070 & 3.55 \\
\hline 16 & -4.670114 & -3.896057 & 4.091537 & -0.086632 & 0.070 & 3.55 \\
\hline 16 & -3.426683 & -4.353531 & 3.621123 & -0.080616 & 0.070 & 3.55 \\
\hline 16 & -2.791848 & -3.739073 & 2.550337 & -0.064599 & 0.070 & 3.55 \\
\hline 27 & -5.889108 & -0.02885 & 1.891704 & -0.537145 & 0.170 & 3.25 \\
\hline 16 & -5.667628 & 1.141637 & 1.284035 & 0.372602 & 0.070 & 3.55 \\
\hline 27 & -4.737211 & 1.294852 & 0.303131 & -0.482006 & 0.170 & 3.25 \\
\hline 16 & -4.276693 & 2.574909 & 0.188462 & 0.505882 & 0.070 & 3.55 \\
\hline 16 & -5.180665 & 3.38776 & 0.994646 & -0.107776 & 0.070 & 3.55 \\
\hline 16 & -6.048588 & 2.49296 & 1.678474 & 0.022993 & 0.070 & 3.55 \\
\hline 16 & -6.978168 & 2.96945 & 2.602802 & -0.128495 & 0.070 & 3.55 \\
\hline 16 & -7.047926 & 4.339814 & 2.813092 & -0.044725 & 0.070 & 3.55 \\
\hline 16 & -6.189203 & 5.224687 & 2.137673 & -0.106283 & 0.070 & 3.55 \\
\hline 16 & -5.241272 & 4.760228 & 1.236011 & -0.052890 & 0.070 & 3.55 \\
\hline 27 & -3.074239 & 2.863942 & -0.311754 & -0.600334 & 0.170 & 3.25 \\
\hline 16 & -9.990221 & -1.958186 & -2.468154 & 0.449143 & 0.070 & 3.55 \\
\hline 16 & -10.478356 & -2.673615 & -1.196773 & -0.179315 & 0.066 & 3.50 \\
\hline 16 & 14.294957 & -1.878811 & -0.02908 & 0.671754 & 0.105 & 3.75 \\
\hline 58 & 15.100194 & -1.155764 & -0.823853 & -0.566056 & 0.170 & 3.00 \\
\hline 16 & -10.96072 & -0.813049 & -2.775334 & -0.177828 & 0.066 & 3.50 \\
\hline 16 & -10.020075 & -2.943964 & -3.648842 & -0.124499 & 0.066 & 3.50 \\
\hline 58 & 14.708809 & -2.780221 & 0.665341 & -0.553645 & 0.210 & 2.96 \\
\hline 61 & -2.951212 & -5.203551 & 4.112895 & 0.098350 & 0.000 & 0.00 \\
\hline 61 & -1.818753 & -4.080606 & 2.196531 & 0.095054 & 0.000 & 0.00 \\
\hline 61 & -6.262919 & -2.444463 & 3.876458 & 0.104702 & 0.000 & 0.00 \\
\hline 61 & -4.562174 & 5.443552 & 0.725486 & 0.094044 & 0.000 & 0.00 \\
\hline 61 & -6.267404 & 6.295416 & 2.332249 & 0.102676 & 0.000 & 0.00 \\
\hline 61 & -7.629881 & 2.279356 & 3.13921 & 0.112173 & 0.000 & 0.00 \\
\hline 61 & 1.238361 & -0.937923 & -0.147169 & 0.120946 & 0.000 & 0.00 \\
\hline 61 & 2.28156 & 3.205168 & -0.773607 & 0.115662 & 0.000 & 0.00 \\
\hline 61 & -0.123582 & 3.844646 & -0.86866 & 0.108844 & 0.000 & 0.00 \\
\hline 61 & -5.436575 & -2.694409 & -1.542868 & 0.078987 & 0.000 & 0.00 \\
\hline 61 & -6.621585 & 1.367635 & -2.223663 & 0.090076 & 0.000 & 0.00 \\
\hline 61 & -7.759035 & -3.437617 & -1.888433 & 0.129632 & 0.000 & 0.00 \\
\hline 61 & -8.946476 & 0.643191 & -2.572156 & 0.109974 & 0.000 & 0.00 \\
\hline 61 & -11.973903 & -1.216976 & -2.920239 & 0.026458 & 0.000 & 0.00 \\
\hline 61 & -11.007524 & -0.08457 & -1.952138 & 0.020038 & 0.000 & 0.00 \\
\hline 61 & -10.682781 & -0.275481 & -3.694198 & 0.028497 & 0.000 & 0.00 \\
\hline 61 & -9.683937 & -2.456196 & -4.576669 & -0.002906 & 0.000 & 0.00 \\
\hline 61 & -9.370759 & -3.813548 & -3.471055 & 0.010130 & 0.000 & 0.00 \\
\hline 61 & -11.043733 & -3.317502 & -3.810086 & 0.015621 & 0.000 & 0.00 \\
\hline 61 & -9.845241 & -3.536993 & -0.944911 & 0.010421 & 0.000 & 0.00 \\
\hline 61 & -10.47374 & -1.989309 & -0.334537 & 0.016160 & 0.000 & 0.00 \\
\hline 61 & -11.507017 & -3.042138 & -1.335983 & 0.036062 & 0.000 & 0.00 \\
\hline 61 & 5.650828 & -1.885537 & -0.167334 & 0.137031 & 0.000 & 0.00 \\
\hline 61 & 8.046107 & -2.389826 & -0.108073 & 0.120412 & 0.000 & 0.00 \\
\hline 61 & 10.793757 & 0.781123 & -1.589706 & 0.137339 & 0.000 & 0.00 \\
\hline 61 & 9.887799 & -2.295024 & 1.28476 & 0.120129 & 0.000 & 0.00 \\
\hline 61 & 13.17627 & 0.124868 & -1.533468 & 0.103301 & 0.000 & 0.00 \\
\hline
\end{tabular}




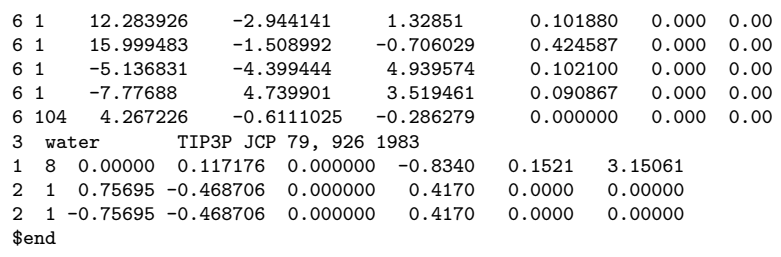

\begin{tabular}{|c|c|c|c|c|c|c|c|c|c|c|c|c|c|c|c|c|c|c|c|c|c|}
\hline \$atoms & fragments & & & & & & & & & & & & & & & & & & & & \\
\hline 11 & 58 & 6 & 90 & 5 & 88 & 4 & & 7 & & 3 & & 87 & & 2 & $89 \mathrm{R}$ & & & & & & \\
\hline 27 & 4 & 8 & 86 & 9 & 85 & 10 & & 16 & & 11 & & 15 & & 14 & 13 & & $12 \mathrm{R}$ & & & & \\
\hline 316 & 10 & 94 & 17 & 18 & 19 & & 69 & & 20 & & 26 & & 35 & & 36 & 37 & 44 & 43 & 63 & 42 & 92 \\
\hline 4 & 28 & 29 & $27 \mathrm{~F}$ & & & & & & & & & & & & & & & & & & \\
\hline 529 & 28 & 30 & 72 & 31 & 74 & & 32 & & 56 & & 33 & & 75 & & 34 & $73 \mathrm{R}$ & & & & & \\
\hline 656 & 57 & 60 & 61 & $32 \mathrm{R}$ & & & & & & & & & & & & & & & & & \\
\hline 757 & 56 & 83 & 82 & 84 & $\mathrm{R}$ & & & & & & & & & & & & & & & & \\
\hline 858 & 59 & 1 & 62 & $\mathrm{~F}$ & & & & & & & & & & & & & & & & & \\
\hline 959 & 58 & 91 & $\mathrm{~F}$ & & & & & & & & & & & & & & & & & & \\
\hline 1060 & 56 & 77 & 76 & $78 \mathrm{R}$ & & & & & & & & & & & & & & & & & \\
\hline 1161 & 56 & 80 & 81 & $79 \mathrm{R}$ & & & & & & & & & & & & & & & & & \\
\hline
\end{tabular}

\$fragment connection

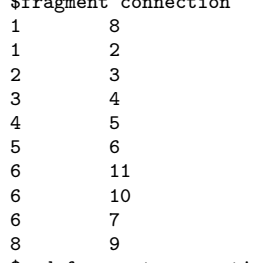

\$end fragment connection

\$bond

$\begin{array}{llll}16 & 0.0 & 0.0\end{array}$

$\begin{array}{lllll}1 & 2 & 0.0 & 0.0\end{array}$

$\begin{array}{llll}2 & 3 & 0.0 & 0.0 \\ 3 & 87 & 0.0 & 0.0\end{array}$

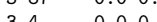

$47 \quad 0.00 .0$

$\begin{array}{llll}45 & 0.0 & 0.0\end{array}$

$\begin{array}{llll}588 & 0.0 & 0.0\end{array}$

$\begin{array}{llll}5 & 6 & 0.0 & 0.0\end{array}$

$\begin{array}{llll}690 & 0.0 & 0.0\end{array}$

$\begin{array}{lllll}7 & 12 & 0.0 & 0.0\end{array}$

$\begin{array}{llll}78 & 0.0 & 0.0\end{array}$

$\begin{array}{lllll}8 & 86 & 0.0 & 0.0\end{array}$

$\begin{array}{llll}89 & 0.0 & 0.0\end{array}$

$\begin{array}{llll}9 & 85 & 0.0 & 0.0\end{array}$

$\begin{array}{llll}910 & 0.0 & 0.0\end{array}$

$\begin{array}{llllllllllll}10 & 16 & 0.0 & 0.0\end{array}$

$\begin{array}{lllll}10 & 11 & 0.0 & 0.0\end{array}$

$\begin{array}{lllll}11 & 15 & 0.0 & 0.0\end{array}$

$\begin{array}{lllllllllllll}11 & 12 & 0.0 & 0.0\end{array}$

$\begin{array}{lllll}12 & 13 & 0.0 & 0.0\end{array}$

$\begin{array}{llll}13 & 14 & 0.0 & 0.0\end{array}$

$\begin{array}{llll}14 & 15 & 0.0 & 0.0\end{array}$

$\begin{array}{llll}1694 & 0.0 & 0.0\end{array}$

$\begin{array}{llll}16 & 17 & 0.0 & 0.0\end{array}$

$\begin{array}{llll}17 & 18 & 0.0 & 0.0\end{array}$

$\begin{array}{lllll}18 & 23 & 0.0 & 0.0\end{array}$

$\begin{array}{lllll}18 & 19 & 0.0 & 0.0\end{array}$

$\begin{array}{llll}19 & 69 & 0.0 & 0.0\end{array}$

$\begin{array}{llll}19 & 20 & 0.0 & 0.0\end{array}$

$\begin{array}{lllll}20 & 26 & 0.0 & 0.0\end{array}$

$\begin{array}{llll}20 & 21 & 0.0 & 0.0\end{array}$

$\begin{array}{lllll}21 & 24 & 0.0 & 0.0\end{array}$

$\begin{array}{lllll}21 & 22 & 0.0 & 0.0\end{array}$

$\begin{array}{llll}22 & 71 & 0.0 & 0.0\end{array}$

$\begin{array}{llll}22 & 23 & 0.0 & 0.0\end{array}$

$\begin{array}{llll}23 & 70 & 0.0 & 0.0\end{array}$

$\begin{array}{llll}24 & 55 & 0.0 & 0.0\end{array}$

$\begin{array}{llll}24 & 25 & 0.0 & 0.0\end{array}$

$\begin{array}{lllll}25 & 27 & 0.0 & 0.0\end{array}$

$\begin{array}{llll}25 & 26 & 0.0 & 0.0\end{array}$

$\begin{array}{llll}26 & 35 & 0.0 & 0.0\end{array}$

$\begin{array}{llll}27 & 47 & 0.0 & 0.0\end{array}$ 


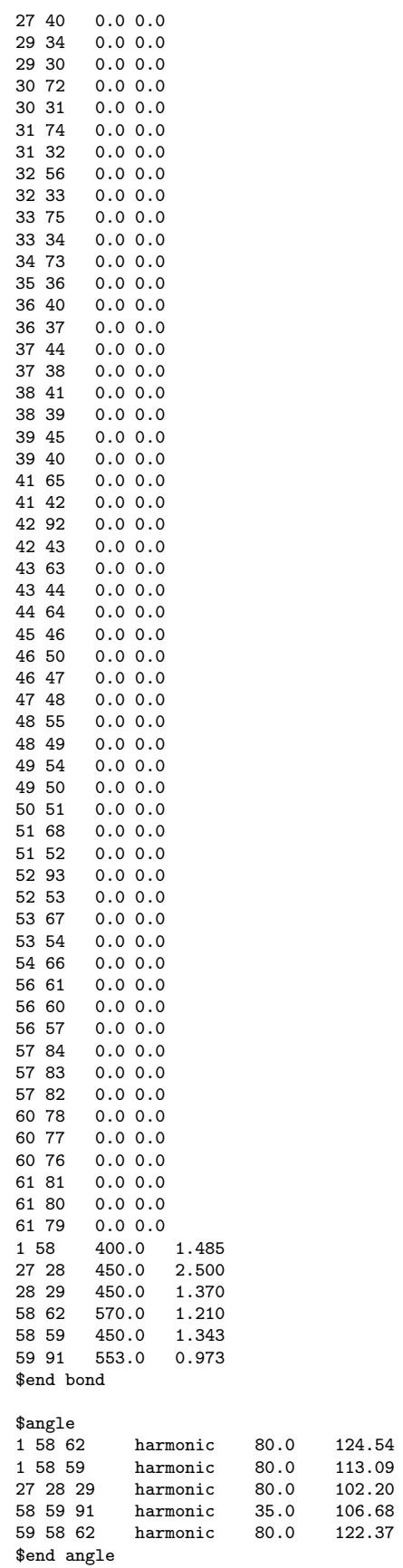

\begin{tabular}{|c|c|c|c|c|c|c|c|}
\hline & & & & & & & \\
\hline 5478 & OPLS & -0.025 & 1.163 & 0.00 & 0.0 & 0.00 .0 & \\
\hline $\begin{array}{llll}5 & 4 & 7 & 12\end{array}$ & OPLS & -0.025 & 1.163 & 0.00 & 0.0 & $0.0 \quad 0.0$ & \\
\hline $\begin{array}{llll}3 & 4 & 7 & 12\end{array}$ & OPLS & -0.025 & 1.163 & 0.00 & 0.0 & 0.00 .0 & \\
\hline 3478 & OPLS & -0.025 & 1.163 & 0.00 & 0.0 & 0.00 .0 & \\
\hline $\begin{array}{llll}6 & 1 & 58 & 62\end{array}$ & OPLS & -0.200 & 4.350 & 0.00 & 0.0 & $0.0 \quad 0.0$ & \\
\hline $\begin{array}{llll}6 & 1 & 58 & 59\end{array}$ & OPLS & 0.000 & 0.000 & 0.00 & 0.0 & 0.00 .0 & \\
\hline 2115862 & OPLS & -0.200 & 4.350 & 0.00 & 0.0 & 0.00 .0 & \\
\hline $\begin{array}{llll}2 & 1 & 58 & 59\end{array}$ & OPLS & 0.000 & 0.000 & 0.00 & 0.0 & 0.00 .0 & \\
\hline $\begin{array}{llll}1 & 58 & 59 & 91\end{array}$ & OPLS & 0.000 & 4.830 & 0.00 & 0.0 & 0.00 .0 & \\
\hline $\begin{array}{llll}62 & 58 & 59 & 91\end{array}$ & OPLS & 0.000 & 4.800 & -3.00 & 0.0 & 0.00 .0 & \\
\hline $\begin{array}{llll}9 & 10 & 16 & 94\end{array}$ & OPLS & 0.000 & 1.100 & 0.00 & 0.0 & 0.00 .0 & \\
\hline $\begin{array}{llll}11 & 10 & 16 & 94\end{array}$ & OPLS & 0.000 & 0.00 & 0.00 & 0.0 & 0.00 .0 & \\
\hline $\begin{array}{llll}25 & 27 & 28 & 29\end{array}$ & OPLS & -0.600 & & 1.200 & 0.90 & $0.0 \quad 0.0$ & \\
\hline $\begin{array}{llll}40 & 27 & 28 & 29\end{array}$ & OPLS & 0.000 & & 0.000 & 0.00 & 0.0 & 0.00 .0 \\
\hline
\end{tabular}


$\begin{array}{lllll}47 & 27 & 28 & 29 & \text { OPLS }\end{array}$

$\begin{array}{lllllll}0.000 & 0.000 & 0.00 & 0.0 & & 0.0 & 0.0 \\ 0.000 & -3.050 & & 0.00 & 0.0 & 0.0 & \end{array}$

$\begin{array}{lllll}27 & 28 & 29 & 34 & \text { OPLS }\end{array}$

$\begin{array}{lllllr}0.000 & -3.050 & 0.00 & 0.0 & 0.0 & 0.0\end{array}$

$\begin{array}{lllll}7 & 28 & 29 & 30 & \text { OPLS }\end{array}$

$\begin{array}{llllll}0.000 & -3.050 & 0.00 & 0.0 & 0.0 & 0.0\end{array}$

$\begin{array}{llll} & -1\end{array}$

$\begin{array}{lllll}31 & 32 & 56 & 60 & \text { OPLS }\end{array}$

$\begin{array}{llll}31 & 32 & 56 & 57\end{array}$

$\begin{array}{llll}33 & 32 & 56 & 61\end{array}$

$\begin{array}{llllll}33 & 32 & 56 & 60 & \text { OPLS }\end{array}$

$\begin{array}{lllllll}0.000 & 80.000 & 0.00 & 0.0 & 0.0 & 0.0\end{array}$

$\begin{array}{llllll}0.000 & 80.000 & 0.00 & 0.0 & 0.0 & 0.0\end{array}$

$\begin{array}{llllll}0.000 & 80.000 & 0.00 & 0.0 & 0.0 & 0.0\end{array}$

$\begin{array}{llllll}0.000 & 80.000 & 0.00 & 0.0 & 0.0 & 0.0\end{array}$

$\begin{array}{llllll}0.000 & 80.000 & 0.00 & 0.0 & 0.0 & 0.0 \\ 0.000 & 80.000 & 0.00 & 0.0 & 0.0 & 0.0\end{array}$

(1)

$\begin{array}{lllll}32 & 56 & 57 & 82 & \text { OPLS }\end{array}$

$\begin{array}{llll}0.0 & 0.0 & 0.46 & 0.0 \\ 0.0 & 0.0 & 0.46 & 0.0\end{array}$

$\begin{array}{llll}0.0 & 0.0 & 0.46 & 0.0\end{array}$

565784 PPIS

$0.46 \quad 0.0$

$\begin{array}{ll}0.46 & 0.0\end{array}$

$\begin{array}{ll}0.46 & 0.0\end{array}$

$\begin{array}{ll}0.46 & 0.0\end{array}$

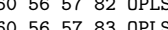

$0.0-0.0$

$\begin{array}{ll}0.46 & 0.0 \\ 0.46 & 0.0\end{array}$

$\begin{array}{ll}0.46 & 0.0 \\ 0.46 & 0.0\end{array}$

$\begin{array}{ll}0.46 & 0.0 \\ 0.46 & 0.0\end{array}$

$\begin{array}{ll}0.46 & 0.0 \\ 0.46 & 0.0\end{array}$

$\begin{array}{ll}0.46 & 0.0\end{array}$

$\begin{array}{ll}0.46 & 0.0\end{array}$

$\begin{array}{lll}0.46 & 0.0\end{array}$

$\begin{array}{ll}0.46 & 0.0\end{array}$

$\begin{array}{ll}0.46 & 0.0\end{array}$

$0.46 \quad 0.0$

$0.46 \quad 0.0$

$\begin{array}{ll}0.46 & 0.0\end{array}$

$0.46 \quad 0.0$

$0.46 \quad 0.0$

$\begin{array}{ll}0.46 & 0.0\end{array}$

$\begin{array}{ll}0.46 & 0.0\end{array}$

$\begin{array}{ll}0.46 & 0.0 \\ 0.46 & 0.0\end{array}$

$\begin{array}{ll}0.46 & 0.0\end{array}$

$\begin{array}{ll}0.46 & 0.0\end{array}$

$\begin{array}{ll}0.46 & 0.0\end{array}$

$0.46 \quad 0.0$

0.0
0.0
0.0

$\begin{array}{ll}0.0 & 0.0 \\ 0.0 & 0.0\end{array}$

$\begin{array}{ll}0.0 & 0.0 \\ 0.0 & 0.0 \\ 0.0 & 0.0\end{array}$

$0.0 \quad 0.0$

$\begin{array}{ll}0.0 & 0.0 \\ 0.0 & 0.0\end{array}$

$\begin{array}{ll}0.0 & 0.0 \\ 0.0 & 0.0\end{array}$

$0.0 \quad 0.0$

$\begin{array}{ll}0.0 & 0.0 \\ 0.0 & 0.0\end{array}$

$0.0 \quad 0.0$

$\begin{array}{ll}0.0 & 0.0 \\ 0.0 & 0.0 \\ 0.0 & 0.0\end{array}$

$\begin{array}{ll}0.0 & 0.0 \\ 0.0 & 0.0\end{array}$

$\begin{array}{ll}0.0 & 0.0 \\ 0.0 & 0.0\end{array}$

$\begin{array}{ll}0.0 & 0.0 \\ 0.0 & 0.0\end{array}$

$\begin{array}{ll}0.0 & 0.0 \\ 0.0 & 0.0\end{array}$

$\begin{array}{lllll}60 & 56 & 61 & 80 & \text { OPLS }\end{array}$

$\begin{array}{ll}0.0 & 0.0 \\ 0.0 & 0.0\end{array}$

0.0

\$improper dihedral

1595862

$15.0 \quad 180.0$

\$end improper dihedral

DICE thermalization input: subpc.ter

title $=$ subpc em agua

ljname $=$ subpc-w.txt

outname $=$ outsubpc $-\mathrm{w}$

ncores $=8$

init $=$ yes

nmol $=12500$

dens $=0.99$

temp $=298.0$

press $=1.0$

upbuf $=150$
accul

accum $=$ no

vstep $=0$

nstep $=20000$

iprint $=1$

isave $=500$

irdf $=0$

iratio $=10$

vratio $=10$

seed $=366045$

dielectric $=80$. 0

\$end

DICE CBMC simulation input: subpc.in

title $=$ subpc em agua

ljname $=$ subpc-w.txt

outname $=$ outsubpc- $\mathrm{w}$

ncores $=8$

init $=$ no

press $=1.0$

upbuf $=150$

accum $=$ no

vstep $=500000$

nstep $=4$

iprint $=1$

isave $=50$

$\operatorname{irdf}=0$

iratio $=10$ 
vratio $=10$

seed $=366045$

sampling $=2$

dielectric $=80.0$

flex $=$ subpc

equiphi $=$ yes

ntrialphi $=32$
fudgel $j=0.5$

fudgel $\mathrm{j}=0.5$
fudgeclb $=0.5$

pcbmc $=0.8$

nsf $=10000$

sfint $=1000$

\$end 


\section{SI 5: References}

[1] Makoto Matsumoto and Takuji Nishimura. Mersenne twister: A 623-dimensionally equidistributed uniform pseudo-random number generator. In ACM Transactions on Modeling and Computer Simulation, volume 8, pages 3-30, 1998.

[2] Mersenne Twister in FORTRAN. http://www.math.sci.hiroshima-u.ac.jp/ $\sim$ m-mat/MT/VERSIONS/FORTRAN/fortran.html. Accessed: 2020-03-25.

[3] M. P. Allen and D. J. Tildesley. Computer Simulation of Liquids, 2nd edition. Oxford University Press, Oxford, 2017.

[4] John C. Owicki. Optimization of Sampling Algorithms in Monte Carlo Calculations on Fluids. pages 159-171. jun 1978.

[5] Bernard Bigot and William L. Jorgensen. Sampling methods for Monte Carlo simulations of n-butane in dilute solution. J. Chem. Phys., 75(4):1944-1952, 1981.

[6] William H. Press, Saul A. Teukolsky, William T. Vetterling, and Brian P. Flannery. Numerical Recipes 3rd Edition: The Art of Scientific Computing. Cambridge University Press, New York, 3rd edition, 2007.

[7] Daan Frenkel and Berend Smit. Understanding molecular simulation. Academic Press, San Diego, 2nd edition, 2002.

[8] William L. Jorgensen, David S. Maxwell, and Julian Tirado-Rives. Development and testing of the OPLS all-atom force field on conformational energetics and properties of organic liquids. J. Am. Chem. Soc., 118(45):11225-11236, 1996.

[9] Wendy D. Cornell, Piotr Cieplak, Christopher I. Bayly, Ian R. Gould, Kenneth M. Merz, David M. Ferguson, David C. Spellmeyer, Thomas Fox, James W. Caldwell, and Peter A. Kollman. A second generation force field for the simulation of proteins, nucleic acids, and organic molecules. J. Am. Chem. Soc., 117(19):5179-5197, 1995.

[10] J.A. Barker and R.O. Watts. Monte Carlo studies of the dielectric properties of waterlike models. Mol. Phys., 26(3):789-792, 1973.

[11] Dominik Sidler, Simon Frasch, Michael Cristòfol-Clough, and Sereina Riniker. Anisotropic reaction field correction for long-range electrostatic interactions in molecular dynamics simulations. J. Chem. Phys., 148(23):234105, 2018.

[12] Sander Pronk, Szilárd Páll, Roland Schulz, Per Larsson, Pär Bjelkmar, Rossen Apostolov, Michael R. Shirts, Jeremy C. Smith, Peter M. Kasson, David VAN DER SPOEL, Berk Hess, and Erik Lindahl. Gromacs 4.5: a high-throughput and highly parallel open source molecular simulation toolkit. Bioinf., 29(7):845-854, 2013. 